\title{
Comparison of experimentally and theoretically determined radiation characteristics of photosynthetic microorganisms
}

\author{
Razmig Kandilian ${ }^{a}$, Jérémy Pruvost ${ }^{\mathrm{a}}$, Arnaud Artu ${ }^{\mathrm{a}}$, Camille Lemasson ${ }^{\mathrm{a}}$, \\ Jack Legrand ${ }^{\mathrm{a}}$, Laurent Pilon ${ }^{\mathrm{b}, *}$ \\ a Université de Nantes, CNRS, GEPEA, UMR-CNRS 6144, Bd de l'Université, CRTT-BP 406, 44602 Saint-Nazaire Cedex, France \\ ${ }^{\mathrm{b}}$ Mechanical and Aerospace Engineering Department, Henry Samueli School of Engineering and Applied Science, University of California, \\ Los Angeles, CA 90095, USA
}

\begin{abstract}
A B S T R A C T
This paper aims to experimentally and directly validate a recent theoretical method for predicting the radiation characteristics of photosynthetic microorganisms. Such predictions would facilitate light transfer analysis in photobioreactors (PBRs) to control their operation and to maximize their production of biofuel and other high-value products. The state of the art experimental method can be applied to microorganisms of any shape and inherently accounts for their non-spherical and heterogeneous nature. On the other hand, the theoretical method treats the microorganisms as polydisperse homogeneous spheres with some effective optical properties. The absorption index is expressed as the weighted sum of the pigment mass absorption cross-sections and the refractive index is estimated based on the subtractive Kramers-Kronig relationship given an anchor refractive index and wavelength. Here, particular attention was paid to green microalgae Chlamydomonas reinhardtii grown under nitrogen-replete and nitrogen-limited conditions and to Chlorella vulgaris grown under nitrogen-replete conditions. First, relatively good agreement was found between the two methods for determining the mass absorption and scattering cross-sections and the asymmetry factor of both nitrogen-replete and nitrogen-limited $C$. reinhardtii with the proper anchor point. However, the homogeneous sphere approximation significantly overestimated the absorption cross-section of $C$. vulgaris cells. The latter were instead modeled as polydisperse coated spheres consisting of an absorbing core containing pigments and a non-absorbing but strongly refracting wall made of sporopollenin. The coated sphere approximation gave good predictions of the experimentally measured integral radiation characteristics of $C$. vulgaris. In both cases, the homogeneous and coated sphere approximations predicted resonance in the scattering phase function that were not observed experimentally. However, these approximations were sufficiently accurate to predict the fluence rate and local rate of photon absorption in PBRs.
\end{abstract}

\section{Introduction}

Microalgal biomass has long been touted as the feedstock for next generation biofuels such as biodiesel. They can also be used for the production of high-value products such as pharmaceuticals, pigments, and nutraceuticals [1]. 
However, technical and economic feasibility of large-scale outdoor production has not yet been fully demonstrated [2]. Microalgae are typically grown in photobioreactors (PBRs) exposed to artificial or solar radiation. The cells use the energy absorbed in the photosynthetically active radiation (PAR) region of the incident solar radiation to carry out photosynthesis. Numerous kinetic models have been developed for coupling microalgae biomass and/or lipid productivity to light transfer in the PBR [3-8]. Such predictive models are crucial for optimizing and for scaling-up bioprocesses to industrial scales as well as for performing economic and life cycle analysis. They require the knowledge of the local fluence rate in the PBR and therefore the solution to the radiative transfer equation (RTE) [9]. The latter depends on the radiation characteristics of the microorganisms including their absorption and scattering cross-sections and their scattering phase function [10]. These radiation characteristics can be obtained experimentally [11] or predicted theoretically based on electromagnetic wave theory [12,13].

The experimental method that we have used for various microorganisms [10,11] applies to absorbing and scattering particles or cells of any arbitrary shape. It assumes that they are (i) dilute to ensure that single scattering prevails, (ii) randomly oriented such that the suspension average scattering phase function has azimuthal symmetry, and (iii) scattering is mainly in the forward direction, as satisfied by cells whose size is much larger than the incident light wavelength. The validity of these assumptions can be verified experimentally $[10,11]$. However, the experimental setup can be costly and the experimental procedure time consuming. Thus, it may be difficult to implement in actual production systems. In addition, measurements are valid only for specific growth conditions and need to be repeated each time conditions change including $\mathrm{pH}$, temperature, illumination, and medium composition. This is particularly true under nitrogen-limited conditions when pigment concentrations decreases dramatically $[14,15]$ and radiation characteristics evolve rapidly with time [16-18].

On the other hand, most theoretical methods predicting the radiation characteristics of microalgae approximated as volume-equivalent spheres and based on Lorenz-Mie theory $[12,13,19,20]$. Others have treated microalgae or cyanobacteria as volume and average projected area equivalent coated spheres [21,22] or infinitely long cylinders [23]. They all considered the cells to be homogeneous, i.e., the effects of the cell organelles and any other heterogeneities within the cells can be accounted for through an effective complex index of refraction $m_{\lambda}=n_{\lambda}+i k_{\lambda}$ where $n_{\lambda}$ and $k_{\lambda}$ are the cells' effective refraction and absorption indices, respectively. The main challenge of the theoretical approach resides in modeling these effective optical properties as a function of wavelength and of the cell's biochemical composition including water content and pigment concentrations, for example. Theoretical methods are relatively fast and could be used for simulating microalgae growth under various operating conditions affecting cell size and/or pigment concentrations. They can also be coupled with kinetic growth models for the design and model-based control of PBRs, and for, ultimately, maximizing their productivity. However, one such theoretical approach has been only validated indirectly by considering the normal-hemispherical transmittance measurements [13].

The present study aims to directly compare, for the first time, the radiation characteristics of microalgae suspensions measured experimentally and predicted theoretically using the state of the art methods. It considered two different green microalgae species namely Chlamydomonas reinhardtii and Chlorella vulgaris grown under either optimal or nitrogen-limited conditions.

\section{Background}

\subsection{Determination of radiation characteristics}

\subsubsection{Experimental method}

The average spectral mass absorption $\bar{A}_{a b s, \lambda}$ and scattering $\bar{S}_{s c a, \lambda}$ cross-sections of dilute suspensions of randomly oriented microorganisms can be experimentally measured according to a method outlined and reviewed by Pilon et al. [10]. First, the scattering phase function can be measured by a polar nephelometer described in detail in Ref. [24]. Then, the apparent extinction coefficient $\beta_{\lambda}^{*}$ of dilute microalgae suspensions with different known mass concentrations $X$ placed in a cuvette, of pathlength $\ell$ can be obtained from normal-normal transmittance $T_{n n, \lambda, X}$ measurements according to [10]

$\beta_{\lambda}^{*}=-\frac{1}{\ell} \ln \left(\frac{T_{n n, \lambda, X}}{T_{n n, \lambda, r e f}}\right)$.

Here, $T_{n n, \lambda, r e f}$ is the normal-normal transmittance of the medium alone in the cuvette. Similarly, the apparent absorption coefficient $\kappa_{\lambda}^{*}$ can be defined from the normalhemispherical transmittance $T_{n h, \lambda}$ measured using an integrating sphere as [10]

$\kappa_{\lambda}^{*}=-\frac{1}{\ell} \ln \left(\frac{T_{n h, \lambda, X}}{T_{n h, \lambda, r e f}}\right)$.

The apparent extinction coefficient $\beta_{\lambda}^{*}$ can be expressed as a function of the actual absorption $\kappa_{\lambda}$ and scattering $\sigma_{s, \lambda}$ coefficients according to [10]

$\beta_{\lambda}^{*}=\kappa_{\lambda}+\left(1-\epsilon_{n}\right) \sigma_{s, \lambda}$.

Here, $\epsilon_{n}$ represents the fraction of light scattered in the forward direction and detected by the spectrometer. Ideally, $\epsilon_{n}$ is equal to zero so that $\beta_{\lambda}^{*}=\beta_{\lambda}=\kappa_{\lambda}+\sigma_{s, \lambda}$. However, due to the finite size of the acceptance angle of the detector, $\epsilon_{n}$ is larger than zero and is assumed to be constant over the PAR region. It can be estimated from the suspension scattering phase function $\Phi_{T, \lambda}(\Theta)$ according to [10]

$\epsilon_{n}=\frac{1}{2} \int_{0}^{\Theta_{a}} \Phi_{T, \lambda}(\Theta) \sin (\Theta) \mathrm{d} \Theta$

where $\Theta_{a}$ is the half acceptance angle of the detector. The actual extinction coefficient $\beta_{\lambda}=\kappa_{\lambda}+\sigma_{s, \lambda}$ can then be 
determined according to [10]

$\beta_{\lambda}=\frac{\beta_{\lambda}^{*}-\epsilon_{n} \kappa_{\lambda}}{1-\epsilon_{n}}$

Similarly, the apparent absorption coefficient $\kappa_{\lambda}^{*}$ is related to the actual absorption $\kappa_{\lambda}$ and scattering $\sigma_{s, \lambda}$ coefficients as [10]

$\kappa_{\lambda}^{*}=\kappa_{\lambda}+\left(1-\epsilon_{h}\right) \sigma_{s, \lambda}$.

Here, $\epsilon_{h}$ is the fraction of the scattered light detected by the detector. Ideally, when all the scattered light is accounted for, $\epsilon_{h}$ is equal to unity and $\kappa_{\lambda}^{*}=\kappa_{\lambda}$. Moreover, at $\lambda=750 \mathrm{~nm}$ green microalgae are assumed to be nonabsorbing, i.e., $\kappa_{750}=0 \mathrm{~m}^{-1}$. Then, Eqs. (3) and (6) simplify to

$\kappa_{750}^{*}=\left(1-\epsilon_{h}\right) \sigma_{s, 750}$ and $\beta_{750}^{*}=\left(1-\epsilon_{n}\right) \sigma_{s, 750}$.

Combining Eqs. (5)-(7) yields

$\kappa_{\lambda}=\kappa_{\lambda}^{*}-\kappa_{750}^{*}\left(\frac{\beta_{\lambda}^{*}-\kappa_{\lambda}^{*}}{\beta_{750}^{*}-\kappa_{750}^{*}}\right)$ and $\sigma_{s, \lambda}=\frac{\beta_{\lambda}^{*}-\epsilon_{n} \kappa_{\lambda}}{1-\epsilon_{n}}-\kappa_{\lambda}$.

Finally, the average spectral mass absorption $\bar{A}_{a b s, \lambda}$ and scattering $\bar{S}_{s c a, \lambda}$ cross-sections (in $\mathrm{m}^{2} / \mathrm{kg}$ ) of the microalgae suspension of dry mass concentration $X$ (in $\mathrm{kg} / \mathrm{m}^{3}$ ) are defined as [10]

$\bar{A}_{a b s, \lambda}=\kappa_{\lambda} / X$ and $\bar{S}_{s c a, \lambda}=\sigma_{s, \lambda} / X$.

In this method, the pathlength $\ell$ and concentration $X$ of the samples are chosen such that single scattering prevails, i.e., photons undergo at most one scattering event as they travel through the suspension. In practice, the cuvette pathlength must be smaller than the photon mean-free path. Then, $\bar{A}_{a b s, \lambda}$ and $\bar{S}_{s c a, \lambda}$ are independent of concentration $X$. This can be verified experimentally by repeating the measurements for suspensions with different mass concentrations $X$. This experimental procedure has been validated with polystyrene microspheres $2-20 \mu \mathrm{m}$ in diameter and long and randomly oriented glass fibers 15$20 \mu \mathrm{m}$ in diameter [11].

\subsection{Theoretical predictions}

Theoretical predictions of $\bar{A}_{a b s, \lambda}$ and $\bar{S}_{s c a, \lambda}$ can be obtained by Lorenz-Mie theory, for both homogeneous or coated spheres, based on the cell size distribution and on the effective complex index of refraction $m_{\lambda}$ of the microalgae $[12,20,25]$. The Lorenz-Mie theory predicts the absorption $C_{a b s, \lambda}$ and scattering $C_{s c a, \lambda}$ cross-sections (in $\mathrm{m}^{2}$ ) and the scattering phase function $\Phi_{\lambda}\left(r_{e q}, \Theta\right)$ of a homogeneous spherical cell of complex index of refraction $m_{\lambda}=n_{\lambda}+i k_{\lambda}$ and volume-equivalent radius $r_{e q}$ in a nonabsorbing medium of refractive index $m_{m, \lambda}=n_{m, \lambda}$. For polydisperse microalgae cells of known size distribution $f\left(r_{e q}\right)$, the average absorption $\bar{C}_{a b s, \lambda}$ and scattering $\bar{C}_{s c a, \lambda}$ cross-sections can be estimated as [10]

$$
\begin{aligned}
& \bar{C}_{a b s, \lambda}=\int_{0}^{\infty} C_{a b s, \lambda}\left(r_{e q}\right) f\left(r_{e q}\right) \mathrm{d} r_{e q} \text { and } \\
& \bar{C}_{s c a, \lambda}=\int_{0}^{\infty} C_{s c a, \lambda}\left(r_{e q}\right) f\left(r_{e q}\right) \mathrm{d} r_{e q} .
\end{aligned}
$$

It is often more practical to express cross-sections in terms of average mass absorption $\bar{A}_{a b s, \lambda}$ and scattering $\bar{S}_{s c a, \lambda}$ cross-sections $[6,7,9,17,26]$. The latter are related to the absorption $\bar{C}_{a b s, \lambda}$ and scattering $\bar{C}_{s c a, \lambda}$ cross-sections by [12]

$\bar{A}_{a b s, \lambda}=\frac{\bar{C}_{a b s, \lambda}}{V_{32} \rho_{d r y}\left(1-x_{w}\right)} \quad$ and $\quad \bar{S}_{s c a, \lambda}=\frac{\bar{C}_{s c a, \lambda}}{V_{32} \rho_{d r y}\left(1-\chi_{w}\right)}$

where $V_{32}$ (in $\mathrm{m}^{3}$ ) is the Sauter mean diameter of the cell, $\rho_{d r y}$ is the density of the dry biomass (in $\mathrm{kg} / \mathrm{m}^{3}$ ), and $x_{w}$ is the average water fraction in the cells.

In addition, the total scattering phase function $\Phi_{T, \lambda}(\Theta)$ of the polydisperse microorganisms is given by [27]

$\Phi_{T, \lambda}(\Theta)=\frac{\int_{0}^{\infty} C_{s c a, \lambda}\left(r_{e q}\right) \Phi_{\lambda}\left(r_{e q}, \Theta\right) f\left(r_{e q}\right) \mathrm{d} r_{e q}}{\int_{0}^{\infty} C_{s c a, \lambda} f\left(r_{e q}\right) \mathrm{d} r_{e q}}$

The backward scattering fraction $b_{\lambda}$ is defined as the fraction of the radiation scattered backwards and is estimated from the suspension's scattering phase function according to [12]

$b_{\lambda}=\frac{1}{2} \int_{\pi / 2}^{\pi} \Phi_{T, \lambda}(\Theta) \sin \Theta \mathrm{d} \Theta$.

The cells' effective absorption index $k_{\lambda}$ and the refractive index $n_{\lambda}$ must be known in order to obtain the average spectral mass absorption $\bar{A}_{a b s, \lambda}$ and scattering $\bar{S}_{s c a, \lambda}$ crosssections of the microorganisms. Pottier et al. [12] proposed the following expression for the absorption index $k_{\lambda}$

$k_{\lambda}=\frac{\lambda}{4 \pi} \sum_{j} C_{j} E a_{j}=\frac{\lambda}{4 \pi} \rho_{d r y}\left(1-x_{w}\right) \sum_{j} w_{j} E a_{j}$

Here, $C_{j}$ (in $\mathrm{kg} / \mathrm{m}^{3}$ ) is the concentration of pigment " $\mathrm{j}$ " in the cell while $w_{j}=C_{j} / X$ is the pigment concentration on a dry mass basis. Moreover, $E a_{j}$ (in $\mathrm{m}^{2} / \mathrm{kg}$ ) is the specific absorption cross-section of individual pigments Chlorophyll $a$ (Chl $a)$, Chlorophyll $b$ (Chl $b)$, photoprotective carotenoids (PPC), and photosynthetic carotenoids (PSC) as reported by Bidigare et al. [28].

Pottier et al. [12] predicted the radiation characteristics of $C$. reinhardtii using the Lorenz-Mie theory assuming that (i) the cells were spherical, (ii) the absorption index $k_{\lambda}$ was given by Eq. (14), and (iii) the refractive index $n_{\lambda}$ was constant over the PAR and equal to 1.55. Recently, Dauchet et al. [13] relaxed the latter assumption and developed a method to predict the spectral refractive index $n_{\lambda}$ of microalgae cells using the subtractive Kramers-Kronig relation given by $[13$

$n_{\nu}=n_{\nu_{p}}+2 \frac{\left(\nu^{2}-\nu_{p}^{2}\right)}{\pi} P \int_{\nu_{\min }}^{\nu_{\max }} \frac{\nu^{\prime} k_{\nu^{\prime}}}{\left(\nu^{\prime 2}-\nu^{2}\right)\left(\nu^{2}-\nu_{p}^{2}\right)} \mathrm{d} \nu^{\prime}$.

where the spectral absorption index $k_{\lambda}$ or $k_{\nu}$ is given by Eq. (14). Here, $\nu=c / \lambda$ is the frequency of radiation, $c$ is the speed of light in vacuum, and $P$ is the Cauchy principal value. The anchor frequency denoted by $\nu_{p}$ was chosen such that the cells did not absorb at that frequency, i.e., $k_{\nu_{p}}=0$. On the other hand, the value for $n_{\nu_{p}}$ must be known or retrieved experimentally. For example, Dauchet et al. [13] chose the anchor wavelength $\lambda_{p}$ as $820 \mathrm{~nm}$ for $C$. reinhardtii as green microalgae do not absorb at $\lambda \geq 750 \mathrm{~nm}$ [13]. The authors retrieved a value of 1.44 for $C$. 
reinhardtii using an inverse method that minimized the difference between the measured and the predicted normal-hemispherical transmittance at $820 \mathrm{~nm}$. The latter was estimated by solving the RTE using the Monte Carlo method and the predicted radiation characteristics of the microorganisms. Finally, they measured the spectral normal-hemispherical transmittance $T_{n h, \lambda}$, over the PAR, of dense microalgae cultures and compared it with predictions by the Monte Carlo method solving the RTE using the radiation characteristics estimated using the theoretical method. The authors assumed that an agreement between the measured and predictions transmittance $T_{n h, \lambda}$ would validate the accuracy of the theoretically predicted radiation characteristics.

Moreover, Lee et al. [29] retrieved the complex index of refraction of $C$. reinhardtii using an inverse method combining the Lorenz-Mie theory, the measured average spectral mass absorption $\bar{A}_{a b s, \lambda}$ and scattering $\bar{S}_{s c a, \lambda}$ crosssections, and the measured surface area equivalent cell radius distribution $f\left(r_{e q}\right)$. The authors reported a refractive index $n_{\lambda}$ over the PAR region ranging from 1.35 to 1.37 and an absorption index $k_{\lambda}$ ranging from 0 to $7 \times 10^{-3}$.

Finally, Quirantes and Bernard [30] modeled Aureococcus anophagefferens cells as coated spheres with a shell volume fraction of $15 \%$. The inner core and outer coating featured complex index of refraction equal to 1.36 and $1.4+i 0.005$ correspond to the cell's cytoplasm and chloroplast, respectively. The authors compared theoretical predictions of algal bloom reflectance to measurements by a tethered surface radiometer. They found better agreement between measurements and predictions when the cells were modeled as coated spheres compared to when they were modeled as homogeneous spheres with a volume-averaged complex index of refraction. This was attributed to the larger backscattering ratio of the coated spheres compared with homogeneous spheres of the same outer radius. Moreover, Geken et al. [31] recently measured the cell wall thickness $t_{w}$ of $C$. vulgaris to be $130 \pm 20 \mathrm{~nm}$. In addition, Atkinson et al. [32] reported that the cell wall of $C$. vulgaris is typically composed of sporopollenin whose refractive index is constant over the PAR region and equal to 1.48 [33].

\subsection{Light transfer modeling in PBRs}

The local rate of photon absorption (LRPA), $\mathcal{A}_{\lambda}(\boldsymbol{r})$, expressed in $\mu \mathrm{mol}_{h \nu} / \mathrm{kg} \mathrm{s}$ represents the amount of photons absorbed per unit weight of biomass and per unit time at location $\boldsymbol{r}$ in the PBR. It has been used to predict the growth kinetics and biomass or lipid productivities of the PBR $[7,17]$. It depends on the average mass absorption cross-section $\bar{A}_{a b s, \lambda}$ of the species and on the local fluence rate $G_{\lambda}(\boldsymbol{r})$ (in $\mu \mathrm{mol}_{h \nu} / \mathrm{m}^{2} \mathrm{~s}$ ) in the PBR, at wavelength $\lambda$ in the PAR region, according to [9]

$\mathcal{A}_{\lambda}(\boldsymbol{r})=\bar{A}_{a b s, \lambda} G_{\lambda}(\boldsymbol{r})$.

In the case of absorbing and scattering media such as microalgal culture, the local spectral fluence rate $G_{\lambda}(\boldsymbol{r})$ can be obtained by solving the radiative transfer equation [20]. For one-dimensional flat-plate PBRs of thickness $L$ with transparent front $(z=0 \mathrm{~m})$ and back $(z=L)$ windows containing strongly forward scattering microalgae exposed to normally incident spectral radiation flux $q_{\lambda, \text { in }}^{\prime \prime}$ the twoflux approximation predicts the local fluence rate $G_{\lambda}(z)$ at depth $z$ as [12]

$\frac{G_{\lambda}(z)}{q_{\lambda, \text { in }}^{\prime \prime}}=2 \frac{\left(1+\alpha_{\lambda}\right) e^{\delta_{\lambda}(L-z)}-\left(1-\alpha_{\lambda}\right) e^{-\delta_{\lambda}(L-z)}}{\left(1+\alpha_{\lambda}\right)^{2} e^{\delta_{\lambda} L}-\left(1-\alpha_{\lambda}\right)^{2} e^{-\delta_{\lambda} L}}$

where the coefficients $\alpha_{\lambda}$ and $\delta_{\lambda}$ are expressed as [12]

$\alpha_{\lambda}=\sqrt{\frac{\bar{A}_{a b s, \lambda}}{\bar{A}_{a b s, \lambda}+2 b_{\lambda} \bar{S}_{s c a, \lambda}}}$ and $\delta_{\lambda}=X \sqrt{\bar{A}_{a b s, \lambda}\left(\bar{A}_{a b s, \lambda}+2 b_{\lambda} \bar{S}_{s c a, \lambda}\right)}$

The two-flux approximation has been shown to give good predictions of the local fluence rate $G_{\lambda}(z)$ for flat-plate PBR and open ponds $[9,12,34]$.

Finally, the PAR-averaged fluence rate $G_{P A R}(z)$ can be expressed as

$G_{P A R}(z)=\int_{400}^{700} G_{\lambda}(z) d \lambda$.

Similarly, the PAR-averaged LRPA $\mathcal{A}_{P A R}(\boldsymbol{r})$ can be expressed as

$\mathcal{A}_{P A R}(\boldsymbol{r})=\int_{400}^{700} \mathcal{A}_{\lambda}(\boldsymbol{r}) \mathrm{d} \lambda$

\subsection{Normal-hemispherical transmittance}

Let us now consider a homogeneous absorbing, scattering, but non-emitting microalgae suspension of thickness $\ell$ exposed to collimated and normally incident radiation. Radiation transfer in this case can be assumed to be one-dimensional $[25,34]$. The cuvette containing the suspension is assumed to be reflecting and refracting and subject to internal reflection. Then solving the RTE based on the modified two-flux approximation leads to an analytical expression for the spectral normal-hemispherical transmittance $T_{n h, \lambda}$ given by [35]

$T_{n h, \lambda, p r e d}=T_{n h, \lambda}^{0}+\frac{D_{\lambda}}{2}\left[\left(1+\rho_{1, \lambda}\right) e^{-\tau_{t r, \lambda, \ell}}+A_{\lambda} / \zeta_{\lambda}\right]$

where $\tau_{t r, \lambda, \ell}$ is the transport optical thickness defined as $\tau_{t r, \lambda, \ell}=\beta_{t r, \lambda} \ell=\left[\bar{A}_{a b s, \lambda}+\left(1-g_{\lambda}\right) \bar{S}_{s c a, \lambda}\right] X \ell$. Here, $T_{n h, \lambda}^{0}$ is the spectral normal-hemispherical transmittance ignoring multiple scattering and expressed as [27]

$T_{n h, \lambda}^{0}=\frac{\left(1-\rho_{1, \lambda}\right)^{2}}{1-\rho_{1, \lambda} C_{t r, \lambda}} e^{-\tau_{t r, \lambda, \ell}}$

where $\rho_{1, \lambda}$ is the normal-normal reflectivity of the quartz/ air interface given by Fresnel's equations. For an optically smooth surface with normally incident radiation, assuming that the quartz is non-absorbing, i.e., $m_{q, \lambda}=n_{q, \lambda}$ in the PAR region, yields,

$\rho_{1, \lambda}=\frac{\left(n_{q, \lambda}-1\right)^{2}}{\left(n_{q, \lambda}+1\right)^{2}}$

The parameters $A_{\lambda}, B_{\lambda}, C_{t r, \lambda}$, and $D_{\lambda}$ are defined as [35]

$A_{\lambda}=\frac{\left(\gamma_{1, \lambda}-\gamma_{2, \lambda} \rho_{1, \lambda}\right)\left(\varphi_{\lambda} s_{\lambda}+c_{\lambda}\right) e^{-\tau_{t r, \lambda, \ell}}-\left(\gamma_{2, \lambda}-\gamma_{1, \lambda} C_{t r, \lambda}\right)}{\left(1+\varphi_{\lambda}^{2}\right) s_{\lambda}+2 \varphi_{\lambda} c_{\lambda}}$ 
$B_{\lambda}=\frac{\left(\gamma_{1, \lambda}-\gamma_{2, \lambda} \rho_{1, \lambda}\right) e^{-\tau_{t r, \ell}}-\left(\gamma_{2, \lambda}-\gamma_{1, \lambda} C_{t r, \lambda}\right)\left(\varphi_{\lambda} s_{\lambda}+c_{\lambda}\right)}{\left(1+\varphi_{\lambda}^{2}\right) s_{\lambda}+2 \varphi_{\lambda} c_{\lambda}}$

$C_{t r, \lambda}=\rho_{1, \lambda} e^{-2 \tau_{t r, \lambda, \ell}}$

$D_{\lambda}=\frac{\gamma_{\lambda}\left(1-\mu_{c, \lambda}^{2}\right) \chi_{\lambda} \zeta_{\lambda}^{2}}{\zeta_{\lambda}^{2}-1}$

Here, the parameters $\gamma_{\lambda}, \mu_{c, \lambda}, \bar{\gamma}_{\lambda}, \gamma_{1, \lambda}, \gamma_{2, \lambda}, \chi_{\lambda}, \varphi_{\lambda}, \zeta_{\lambda}, s_{\lambda}$, and $c_{\lambda}$ are given by [35]

$$
\begin{aligned}
& \gamma_{\lambda}=\frac{1-\rho_{1, \lambda}}{1+\rho_{1, \lambda}}, \quad \mu_{c, \lambda}=\frac{\left(n_{q, \lambda}^{2}-1\right)^{1 / 2}}{n_{q, \lambda}}, \\
& \bar{\gamma}_{\lambda}=\frac{\gamma_{\lambda}}{1+\mu_{c, \lambda}}, \gamma_{1, \lambda}=1-2 \bar{\gamma}_{\lambda}, \\
& \gamma_{2, \lambda}=1+2 \bar{\gamma}_{\lambda}, \chi_{\lambda}=\frac{\omega_{\mathrm{tr}, \lambda}}{1-\omega_{\mathrm{tr}, \lambda}} \frac{1-\rho_{1, \lambda}}{1-\rho_{1, \lambda} c_{\mathrm{tr}, \lambda}}, \\
& \varphi_{\lambda}=2 \bar{\gamma}_{\lambda} / \zeta_{\lambda}, \zeta_{\lambda}^{2}=\frac{4}{\left(1+\mu_{c, \lambda}\right)^{2}} \frac{1-\omega_{\mathrm{tr}, \lambda}}{1-\omega_{\mathrm{tr}, \lambda} \mu_{c, \lambda}}, \\
& s_{\lambda}=\sinh \left(\zeta_{\lambda} \tau_{\mathrm{tr}, \lambda, \ell}\right), \quad \text { and } \quad c_{\lambda}=\cosh \left(\zeta_{\lambda} \tau_{\mathrm{tr}, \lambda, \ell}\right) .
\end{aligned}
$$

Here, $\mu_{c, \lambda}$ is the director cosine of the critical angle $\theta_{c}$ beyond which total internal reflection occurs while the transport single scattering albedo is defined as $\omega_{t r, \lambda}=\sigma_{t r, \lambda}$ $\beta_{t r, \lambda}$.

The present study directly compares the measured and predicted absorption and scattering cross-sections and the scattering phase function of $C$. reinhardtii, grown under optimal and nitrogen-limited conditions, and C. vulgaris grown under optimal conditions. Then, the spectral normal-hemispherical transmittance of various concentrated microalgae suspensions was measured and compared with predictions by the modified two-flux approximation based on the measured or the theoretically predicted radiation characteristics. Finally, the PAR-averaged fluence rate and local rate of photon absorption (LRPA) of the three cultures grown in the study and estimated using the radiation characteristics determined by both methods were compared.

\section{Materials and methods}

\subsection{Species and culture medium}

A wild type of Chlamydomonas reinhardtii (137 AH) was supplied by Commissariat à l'Energie Atomique (CEA) Cadarache, France while the C. vulgaris (211/19) strain was obtained from the culture collection of protozoa and microalgae (CCAP) in Scotland, UK. Both species were cultivated in a modified Suoeka medium with the following composition (in $\mathrm{mM}$ ): $\mathrm{NH}_{4} \mathrm{Cl} 2.71, \mathrm{MgSO}_{4} \cdot 7 \mathrm{H}_{2} \mathrm{O} 1.14$, $\mathrm{CaCl}_{2} \cdot 2 \mathrm{H}_{2} \mathrm{O} 0.34, \mathrm{KH}_{2} \mathrm{PO}_{4} 4.48, \mathrm{NaHCO}_{3} 20$, and $1 \mathrm{~mL}$ of Hutner's trace elements solution. The medium was sterilized by autoclaving it at $121^{\circ} \mathrm{C}$ for $25 \mathrm{~min}$.

C. reinhardtii culture was grown in optimal conditions in a $1.5 \mathrm{~L}$ torus type PBR with a depth of $4 \mathrm{~cm}$ in chemostat mode with a culture dilution rate of $0.011 / \mathrm{h}$. It was exposed to $110 \mu \mathrm{mol}_{h \nu} / \mathrm{m}^{2} \mathrm{~s}$ of white LED light. A detailed description of the PBR was given by Takache et al. [6] and need not be repeated. The nitrogen-limited $C$. reinhardtii culture was grown in the same torus PBR but in a modified Suoeka medium containing $1 \mathrm{mM}$ of $\mathrm{NH}_{4}$ instead of $2.71 \mathrm{mM}$ and exposed to $400 \mu \mathrm{mol}_{h_{\nu}} / \mathrm{m}^{2} \mathrm{~s}$ white LED light. Finally, C. vulgaris cells were grown in a $3 \mathrm{~cm}$ thick flat plate airlift PBR, described in Ref. [14], and exposed to $500 \mu \mathrm{mol}_{h \nu} / \mathrm{m}^{2} \mathrm{~s}$ white LEDs. This PBR was operated in chemostat mode with a dilution rate of $0.011 / \mathrm{h}$.

Fig. 1 shows micrographs of (a) C. reinhardtii grown in optimal or (b) nitrogen limited conditions and of (c) $C$. vulgaris grown in optimal conditions. The $C$. reinhardtii cells had a radius between 3 and $6 \mu \mathrm{m}$ while $C$. vulgaris was much smaller and had an approximate radius of $2 \mu \mathrm{m}$.

\subsection{Biomass concentration}

Microorganism dry biomass concentration $X$ (in $\mathrm{kg} / \mathrm{m}^{3}$ ) was measured gravimetrically by filtering $5 \mathrm{~mL}$ of culture through a pre-dried and pre-weighed $0.45 \mu \mathrm{m}$ pore size glass-microfiber filter (Whatman GF/F). The filters were dried for a minimum of $24 \mathrm{~h}$ in an oven at $105^{\circ} \mathrm{C}$ and weighed after being cooled in a desiccator for $30 \mathrm{~min}$. Each sample was analyzed in triplicates and the mean value of the biomass concentration was reported.

\subsection{Pigment concentrations}

Photosynthetic pigments were extracted in pure methanol and quantified spectrophotometrically. A volume of $0.5 \mathrm{~mL}$ of culture was first centrifuged at $13,400 \mathrm{rpm}(12,100 \mathrm{~g})$ for $10 \mathrm{~min}$. The medium was discarded and the cells were resuspended in $1 \mathrm{~mL}$ of pure methanol and sonicated for $20 \mathrm{~s}$. The samples were left for $1 \mathrm{~h}$ in an oven at $45^{\circ} \mathrm{C}$ and the extract was then centrifuged. The optical density $\mathrm{OD}_{\lambda}$ of the supernatant was measured at wavelengths $750,665,652$, and $480 \mathrm{~nm}$ using a UV-vis spectrophotometer (Jasco V-730 Easton, MD). All extractions were performed in triplicates. Chlorophyll $a$ and $b$ concentrations, respectively denoted by $C_{\text {Chla }}$ and $C_{C h l b}$, were estimated according to the correlations [36]

$$
\begin{aligned}
C_{\text {Chla }}[\mathrm{mg} / \mathrm{L}]= & -8.0962\left(O D_{652}-O D_{750}\right) \\
& +16.5169\left(O D_{665}-O D_{750}\right) \\
C_{\text {Chlb }}[\mathrm{mg} / \mathrm{L}]= & 27.4405\left(O D_{652}-O D_{750}\right) \\
& -12.1688\left(O D_{665}-O D_{750}\right) .
\end{aligned}
$$

Similarly, the total carotenoid concentration $C_{P P C}+P S C$, accounting for both photoprotective and photosynthetic carotenoids, was estimated according to [37]

$C_{P P C}+P S C[\mathrm{mg} / \mathrm{L}]=4\left(O D_{480}-O D_{750}\right)$.

The ratio of PPC to PSC concentrations of both species was assumed to be 6 for any growth condition [38].

\subsection{Determination of size distribution}

The cell size distribution was measured using 2D microscope images captured using a Zeiss microscope connected to a CCD camera. The image analysis software image J was used to manually measure the major $a$ and minor $b$ Ferret diameters of individual cells. Then, spheroid aspect ratio was defined as $\epsilon=a / b$ while the volume- 

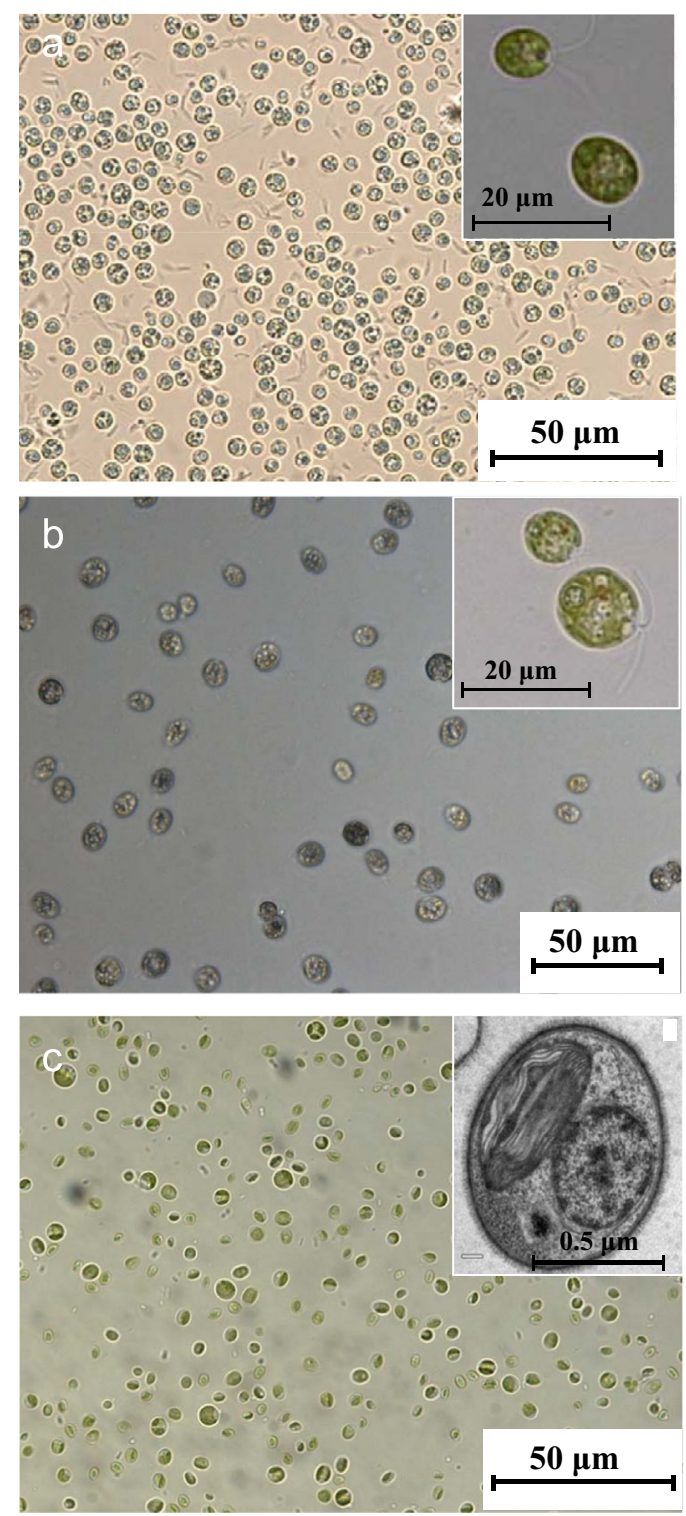

Fig. 1. Micrographs of (a) C. reinhardtii grown in optimal conditions and in (b) nitrogen limited conditions and of (c) C. vulgaris grown in optimal conditions. Inset of (c) shows a transmission electron micrograph of $C$. vulgaris (from Ref. [31] reproduced with permission from Springer).

equivalent radius $r_{e q}$ was determined as [13]

$r_{e q}=a\left(\frac{3}{2 \epsilon}\right)^{1 / 3}$

The frequency distribution of the volume-equivalent radius $f\left(r_{e q}\right)$ was estimated according to

$f\left(r_{e q}\right)=\frac{N\left(r_{e q}\right)}{\int_{0}^{\infty} N\left(r_{e q}\right) \mathrm{d} r_{e q}}=\frac{N\left(r_{e q}\right)}{N_{T}}$

where $N\left(r_{e q}\right)$ is the number of cells per unit volume of suspension with a volume-equivalent radius between $r_{e q}$ and $r_{e q}+\Delta r_{e q}$. Here, $N_{T}$ is total cell concentration in suspension (in cells $/ \mathrm{m}^{3}$ ). A minimum of 500 cells were measured from each culture and the bin size $\Delta r_{e q}$ was set to $0.1 \mu \mathrm{m}$.

\subsection{Radiation characteristics measurements}

The total scattering phase function $\Phi_{T, \lambda}(\Theta)$ was assumed to be independent of radiation wavelength over the PAR region. It was measured at $633 \mathrm{~nm}$ by a polar nephelometer whose experimental setup and data analysis have previously been reported and validated in detail by Berberoğlu et al. [24]. Due to interference of the probe with the laser beam, the scattering phase function could not be measured beyond $160^{\circ}$ with respect to the forward direction.

The normal-normal transmittance $T_{n n, \lambda}$ measurements were performed using a UV-vis-NIR spectrophotometer (Agilent Cary 5000, Santa Clara, CA). The normal-hemispherical transmittance $T_{n h, \lambda}$ measurements were performed using an integrating sphere attachment (Agilent Cary DRA-2500, Santa Clara, CA) to the same spectrophotometer. Here also, the microalgae were centrifuged at $13,400 \mathrm{rpm}(12,100 \mathrm{~g})$ for $10 \mathrm{~min}$ and washed with phosphate buffer saline (PBS) solution and resuspended in PBS to avoid absorption and scattering by the growth medium. The measurements were performed in $1 \mathrm{~cm}$ pathlength quartz cuvettes (110-10-40 Hellma Analytics, Müllheim, Germany) in the wavelength range from 350 to $750 \mathrm{~nm}$. The microalgae suspensions were diluted sufficiently $\left(X \leq 0.1 \mathrm{~kg} / \mathrm{m}^{3}\right)$ to ensure that single scattering prevailed [39]. The average spectral absorption $\bar{A}_{a b s, \lambda}$ and scattering $\bar{S}_{s c a, \lambda}$ cross-sections of microalgae suspensions were measured for three biomass concentrations $X$ between 0.01 and $0.1 \mathrm{~kg} / \mathrm{m}^{3}$ to ensure that they were independent of $X$. The cross-sections reported correspond to the mean of the three measurements and the error bars correspond to $95 \%$ confidence interval.

\subsection{Theoretical method}

The radiation characteristics of microalgae were predicted theoretically based on the method developed by Dauchet et al. [13]. The microorganisms were modeled as homogeneous spheres with an effective complex index of refraction $m_{\lambda}=n_{\lambda}+i k_{\lambda}$ where $k_{\lambda}$ and $n_{\lambda}$ were predicted by Eqs. (14) and (15), respectively. The anchor refractive index $n_{820}$ used in Eq. (15) was taken as either $n_{820}=1.44$ or $n_{820}=1.37$. The former was recommended by Dauchet et al. [13] and the latter corresponded to the refractive index at $750 \mathrm{~nm}$ reported by Lee et al. [29], both for $C$. reinhardtii. These anchor refraction indices were used for $C$. vulgaris since it is also a green microalgae and due to the lack of information available in the literature. The dry biomass density $\rho_{\text {dry }}$ and the water fraction $x_{w}$ of the cells were taken as $1400 \mathrm{~kg} / \mathrm{m}^{3}$ and $0.78 \pm 0.02$, respectively [13].

Moreover, the C. vulgaris cells were also modeled as coated spheres with an absorbing inner core of radius $r_{c}$ and refraction and absorption indices $n_{c, \lambda}$ and $k_{c, \lambda}$ surrounded by a non-absorbing coating (or wall) of thickness $t_{w}$ with refractive index $n_{w, \lambda}$. The Lorenz-Mie theory for coated spheres was used to predict the absorption $C_{a b s, \lambda}$ and scattering $C_{s c a, \lambda}$ cross-sections of the coated sphere 
based on (i) its core radius $r_{c}$, (ii) its wall thickness $t_{w}$, (iii) the core complex index of refraction $m_{c, \lambda}=n_{c, \lambda}+i k_{c, \lambda}$, and (iv) the wall refractive index $n_{w, \lambda}$. Here, the outer radius distribution of the coated sphere was assumed to be equal to the volume-equivalent radius $r_{e q}$ distribution of the homogeneous sphere. Then, the core radius was estimated as $r_{c}=r_{e q}\left(1-t_{w} / \bar{r}_{e q}\right)$ where the mean equivalent radius $\bar{r}_{e q}$ of $C$. vulgaris was reported as $1.89 \mu \mathrm{m}$ [40].

When modeling the cell as a coated sphere with an absorbing core, the effective absorption index of the homogeneous cell $k_{\lambda}$ given by Eq. (14) cannot be used for the core. In order to conserve the mass of pigments in the cell while maintaining the same overall cell volume, the effective absorption index of the core $k_{c, \lambda}$ can be expressed as [12]

$k_{c, \lambda}=\frac{\lambda}{4 \pi} a_{c m, \lambda}$

where $a_{c m, \lambda}$ is the effective absorption coefficient (in $1 / \mathrm{m}$ ) of the substance contained in the microalgae core expressed as [12]

$a_{c m, \lambda}=\sum_{j} E a_{j, \lambda} C_{j, c}$.

Here, $C_{j, c}$ is the pigment concentration in the core (in $\mathrm{kg} /$ $\mathrm{m}^{3}$ ). It can be expressed as $C_{j, c}=m_{j} / V_{c}$ where $m_{j}$ is the average mass of pigment per cell (in $\mathrm{kg} /$ cell). The latter can be estimated by $m_{j}=C_{j} / N_{T}$ where $C_{j}$ is the measured concentration of pigment $j$ in the culture (in $\mathrm{kg} / \mathrm{m}^{3}$ ) and $N_{T}$ is the cell number density in the culture (in cells $/ \mathrm{m}^{3}$ ). It is related to the measured biomass concentration by [12]

$N_{T}=\frac{X}{\rho_{\text {dry }}\left(1-x_{w}\right) V_{e q}}$

Thus, taking into account the fact that $w_{j}=C_{j} / X$, the core absorption index $k_{c, \lambda}$ can be expressed as

$k_{c, \lambda}=\frac{\lambda}{4 \pi} \sum_{j} E a_{j, \lambda} w_{j} \rho_{d r y}\left(1-x_{w}\right) \frac{V_{e q}}{V_{c}}=k_{\lambda} \frac{V_{e q}}{V_{c}}$

Similarly, the refractive index $n_{c, \lambda}$ of the core was determined using Eq. (15) by replacing $k_{\lambda}$ (or $k_{\nu}$ ) with $k_{c, \lambda}\left(\right.$ or $\left.\mathrm{k}_{\mathrm{c}, \nu}\right)$.

In order to predict the normal-hemispherical transmittance $T_{n h, \lambda}$ using Eqs. (21)-(28), one needs to know (i) the thickness $\ell$ of the suspension, (ii) the index of refraction of the quartz cuvette $n_{q, \lambda}$, (iii) the absorption coefficient of the suspension $\kappa_{\lambda}$, and (iv) the transport scattering coefficient $\sigma_{s, t r, \lambda}=\sigma_{s, \lambda}\left(1-g_{\lambda}\right)$. Here, the thickness $\ell$ of the cuvette was $10 \mathrm{~mm}$. The spectral refractive index of the quartz cuvette was given by the dispersion relation [41]

$n_{q, \lambda}^{2}-1=\frac{B_{1} \lambda^{2}}{\lambda^{2}-C_{1}}+\frac{B_{2} \lambda^{2}}{\lambda^{2}-C_{2}}+\frac{B_{3} \lambda^{2}}{\lambda^{2}-C_{3}}$

where the wavelength $\lambda$ is expressed in $\mu \mathrm{m}$ and ranges from 0.2 to $2.5 \mu \mathrm{m}$. The constants $B_{1}, B_{2}, B_{3}, C_{1}, C_{2}$, and $C_{3}$ are respectively equal to $4.73115591 \times 10^{-1}, 6.31038719$ $\times 10^{-1}, \quad 9.06404498 \times 10^{-1}, \quad 1.29957170 \times 10^{-2}$, $4.12809220 \times 10^{-3}$, and $9.87685322 \times 10^{1}$ [41]. On the other hand, in the visible part of the spectrum, quartz can be safely treated as non-absorbing, i.e., $k_{q, \lambda} \approx 0$ [41]. This corresponded to $\mu_{c}$ around 0.73 or to a critical angle $\theta_{c}$ of about $43^{\circ}$ while the reflectivity $\rho_{1, \lambda}$ was about $3.5 \%$.

Finally, the average spectral mass absorption $\bar{A}_{a b s, \lambda}$ and scattering $\bar{S}_{\text {sca }, \lambda}$ cross-sections of a suspension of a polydisperse microorganisms were estimated using Eq. (10). Note that dry biomass density of the coated spheres was assumed to be identical to that of the homogeneous cell $\rho_{\text {dry }}$ and equal to $1400 \mathrm{~kg} / \mathrm{m}^{3}$ as previously discussed. The theoretical predictions of the radiation characteristics of homogeneous or coated sphere microorganisms were implemented in Matlab ${ }^{\circledR}$ and the code was validated against results reported in Ref. [13] for C. reinhardtii treated as homogeneous volume-equivalent spheres.

\section{Results and discussion}

\subsection{Size distribution and pigment concentrations}

Table 1 summarizes the measured mean equivalent radius $\bar{r}_{e q}$ and the Chl $a$, Chl $b$, PPC, and PSC concentrations of $C$. reinhardtii grown under optimal or nitrogen-limited conditions and of $C$. vulgaris grown under optimal conditions. These values were used to predict the radiation characteristics of the microalgae for each growth condition. Moreover, $C$. reinhardtii featured significantly smaller pigment concentrations when they were grown in nitrogen-limited conditions than in optimal conditions. This observation has been made previously for various microorganisms and was attributed to reduction in photosynthetic antenna size as a protection mechanism against light-induced oxidative stress [14,16,17]. Lastly, C. vulgaris grown under optimal conditions featured the largest pigment concentration of the three cultures examined.

Figs. 2(a)-(c) show histograms of the volumeequivalent radius frequency distributions and fitted lognormal distributions $f\left(r_{e q}\right)$ of $C$. reinhardtii grown in optimal and in nitrogen-limited conditions and C. vulgaris grown in optimal conditions. Furthermore, Fig. 2(d) shows

Table 1

Measured mean volume-equivalent radius $\bar{r}_{e q}$ and $\mathrm{Chl} a$, Chl $b$, PPC, and PSC concentrations of $C$. reinhardtii grown under optimal or nitrogen-limited conditions and of $C$. vulgaris grown under optimal conditions.

\begin{tabular}{|c|c|c|c|c|c|c|}
\hline Species & Growth conditions & $\bar{r}_{e q}(\mu \mathrm{m})$ & Chl $a$ (wt\%) & Chl $b$ (wt\%) & PPC (wt\%) & PSC (wt\%) \\
\hline C. reinhardtii & optimal & $4.03 \pm 0.78$ & $3.2 \pm 0.3$ & $1.21 \pm 0.1$ & $0.92 \pm 0.09$ & $0.15 \pm 0.01$ \\
\hline C. reinhardtii & N-limited & $4.09 \pm 0.55$ & $0.17 \pm 0.01$ & $0.07 \pm 0.003$ & $0.12 \pm 0.005$ & $0.02 \pm 0.001$ \\
\hline C. vulgaris & optimal & $1.89 \pm 0.21$ & $3.82 \pm 0.06$ & $1.14 \pm 0.03$ & $0.85 \pm 0.02$ & $0.14 \pm 0.03$ \\
\hline
\end{tabular}


a

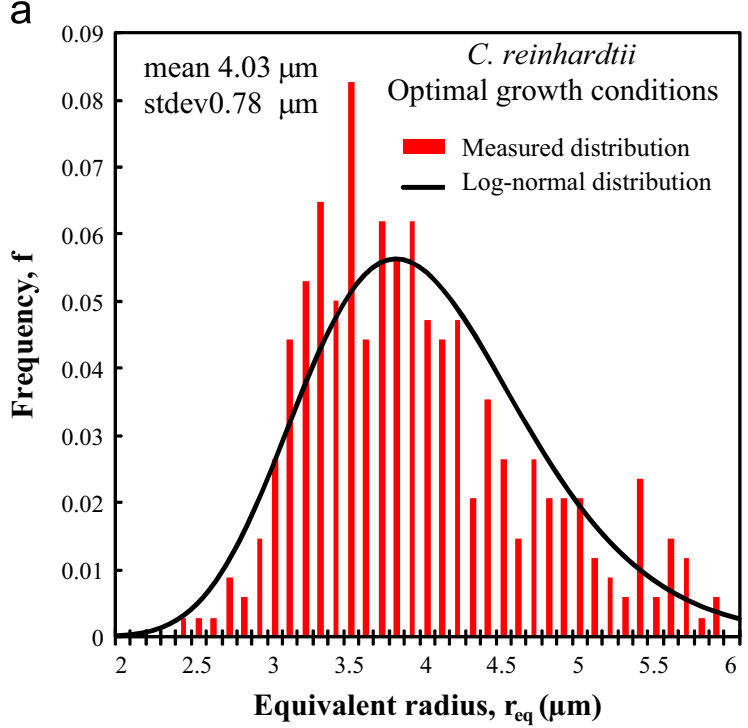

C

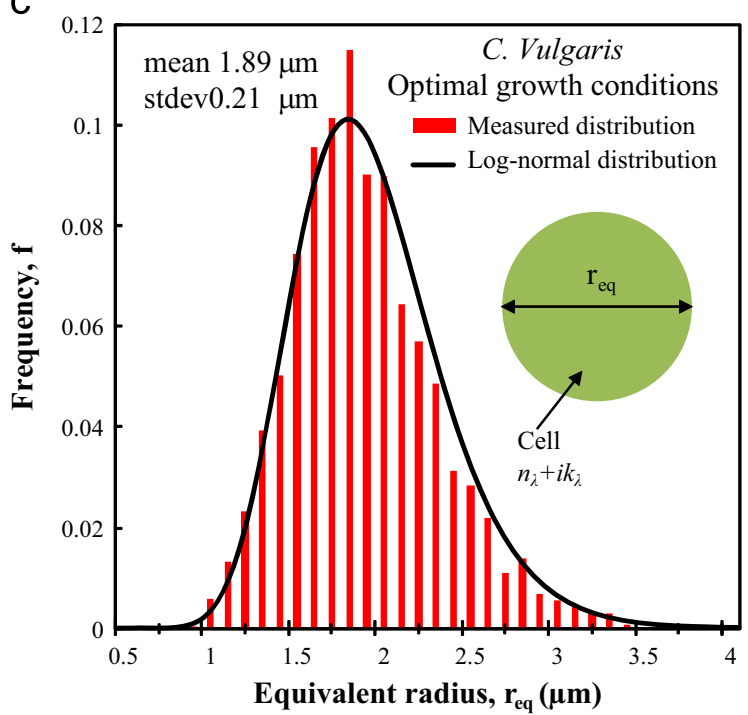

b

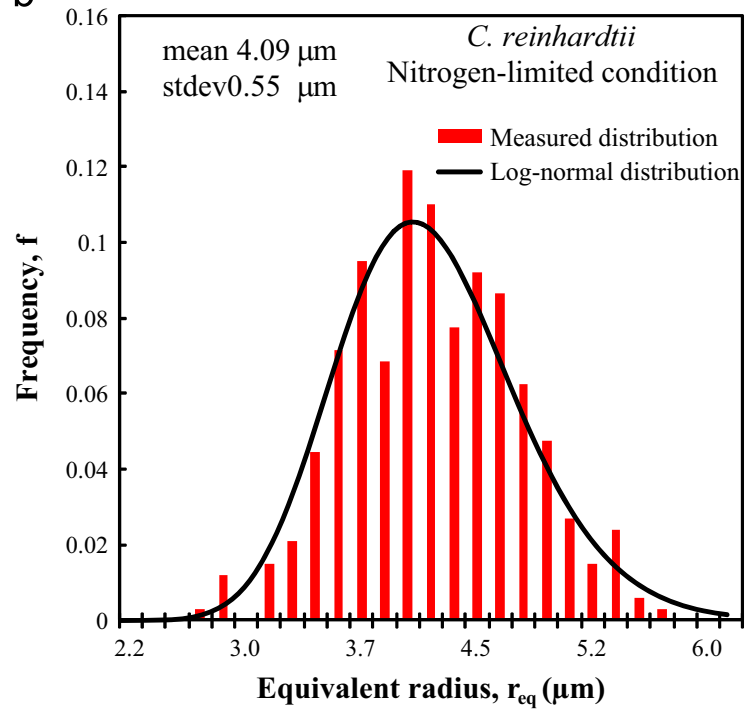

d

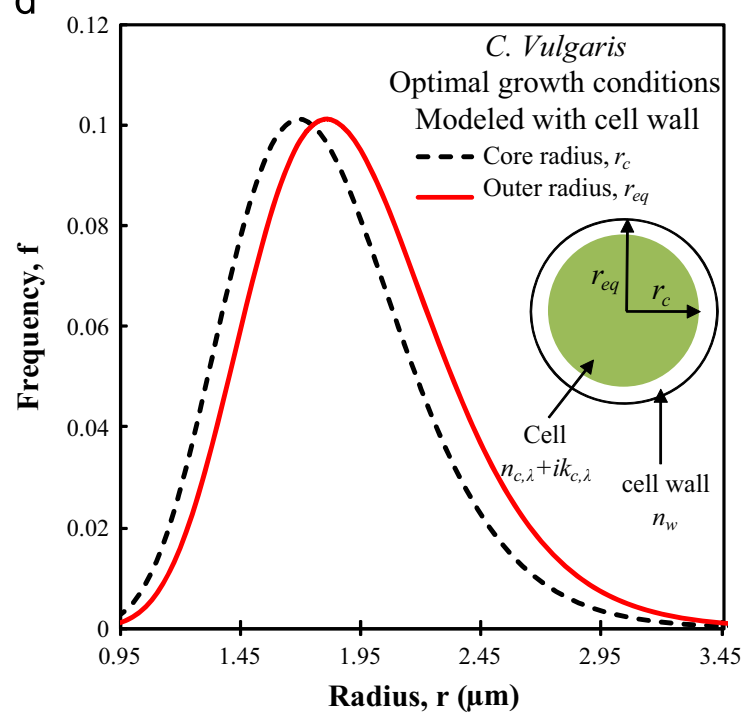

Fig. 2. Measured volume-equivalent radius distribution $f\left(r_{e q}\right)$ and fitted log-normal distribution of (a) C. reinhardtii grown in optimal conditions, (b) C. reinhardtii grown in nitrogen-limited conditions, (c) C. vulgaris modeled as homogeneous spheres, and (d) the log-normal distribution of core $r_{c}$ and outer $r_{e q}$ radii of $C$. vulgaris modeled as coated spheres.

the fitted log-normal distribution of the core $r_{c}$ and outer $r_{e q}$ radii of $C$. vulgaris modeled as coated spheres to represent their refracting cell wall.

The mean volume-equivalent radius $\bar{r}_{e q}$ of $C$. reinhardtii cells grown in optimal and nitrogen-limited conditions were nearly identical and measured as $4.03 \mu \mathrm{m}$ and $4.09 \mu \mathrm{m}$, respectively. This was consistent with the volumeequivalent radius of $3.93 \mu \mathrm{m}$ reported by Dauchet et al. [13] for $C$. reinhardtii grown in optimal conditions. Similarly, Berberoğlu et al. [24] reported the surface area equivalent radius of $C$. reinhardtii as $4.03 \mu \mathrm{m}$. The difference between these reported mean radii was smaller than $2.5 \%$ and may be due to differences in the cultivation conditions. It may also be due to experimental uncertainty and/or differences in the experimental and analytical tools used. On the other hand, $C$. vulgaris cells had a mean volume-equivalent radius $\bar{r}_{e q}$ of $1.89 \mu \mathrm{m}$. Finally, the coated sphere $C$. vulgaris featured outer radius $r_{e q}$ distribution equal to the volume-equivalent homogeneous sphere radius distribution $\mathrm{f}\left(\mathrm{r}_{\mathrm{eq}}\right)$.

\subsection{Scattering phase function}

Figs. 3(a)-(c) compare the experimentally measured and theoretically predicted total scattering phase function $\Phi_{T, 633}(\Theta)$ at $633 \mathrm{~nm}$ of (a) C. reinhardtii grown in optimal and (b) in nitrogen-limited conditions and of (c) C. vulgaris grown in optimal conditions. In all cases, scattering was mostly in the forward direction. This was expected as the cells were much larger than the wavelength of light. Indeed, the measured asymmetry factor $g_{633}$ of $C$. 
a

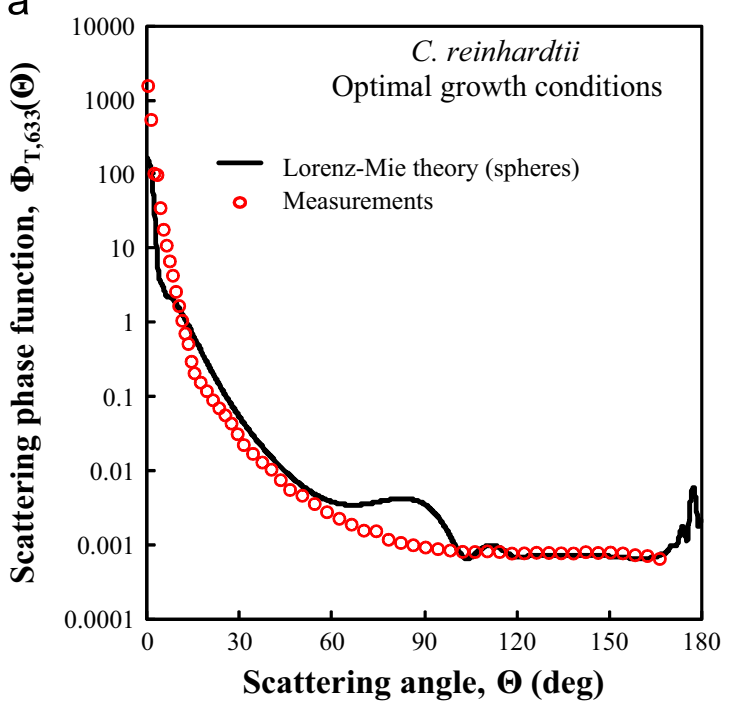

b

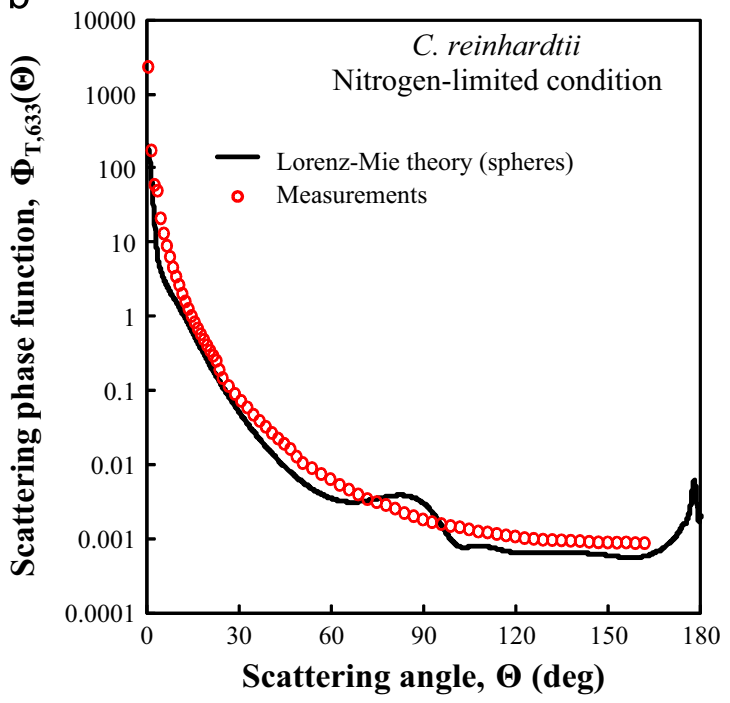

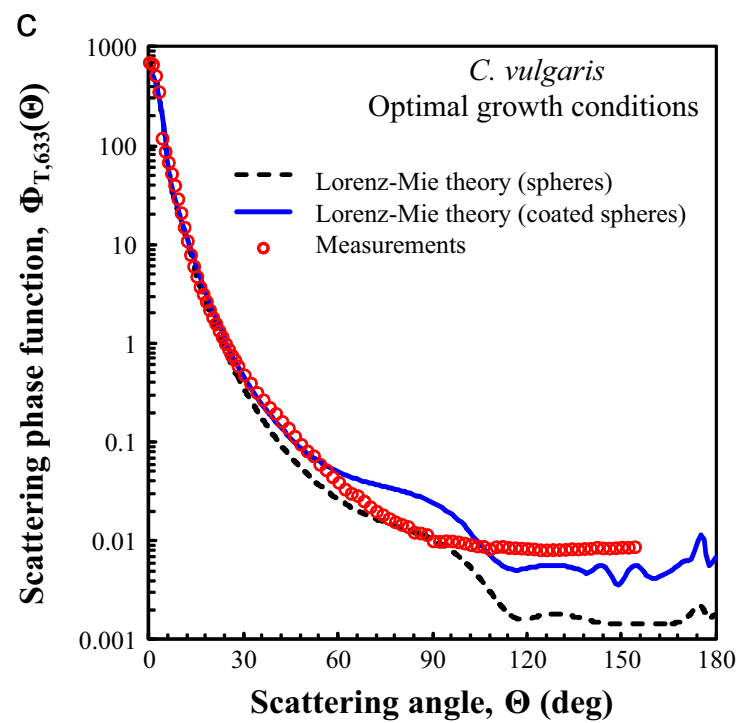

Fig. 3. Experimentally measured and theoretically predicted total scattering phase function $\Phi_{T, 633}(\Theta)$ at 633 nm for $C$. reinhardtii culture grown in (a) optimal and (b) nitrogen-limited conditions, and (c) for C. vulgaris culture grown in optimal conditions.

reinhardtii grown in both optimal and nitrogen-limited conditions was equal to 0.98 while that of $C$. vulgaris was 0.974 . On the other hand, the theoretically predicted asymmetry factor $g_{633}$ obtained by treating the microalgae as homogeneous spheres was estimated as 0.98, 0.962, and 0.982 , respectively. Similarly, the measured backward scattering fraction $b_{633}$ was 0.008 for $C$. reinhardtii grown in either optimal or nitrogen-limited conditions and that of $C$. vulgaris was 0.004 . The corresponding theoretically predicted backward scattering fraction $b_{633}$ was 0.006 , 0.007 , and 0.002 , respectively.

The scattering phase function predicted for $C$. vulgaris modeled as coated spheres agreed better with experimental measurements than when treating them as homogeneous spheres. This may be due to an increase in backward scattering of the coated sphere compared with homogeneous sphere due to the mismatch in the refractive index between the medium, the coating, and the core. Indeed, the predicted backward scattering fraction $b_{633}$ of the coated sphere was two times larger than that of the homogeneous spherical cells and equal to the measured value of $b_{633}=0.004$. Similarly, the predicted asymmetry factor using the coated sphere approximation was 0.972 compared with 0.974 obtained experimentally.

The scattering phase functions predicted theoretically show good agreement with experimental measurements in the forward directions where scattering is dominated by diffraction and is not significantly affected by the choice of the model. However, strong angular resonances were predicted for scattering angles larger than $60^{\circ}$ but were not observed experimentally. This may be the result of modeling heterogeneous non-spherical cells as homogeneous or coated spheres as heterogeneities destroy the angular resonances [42-44]. More advanced models such as the multi- 
a

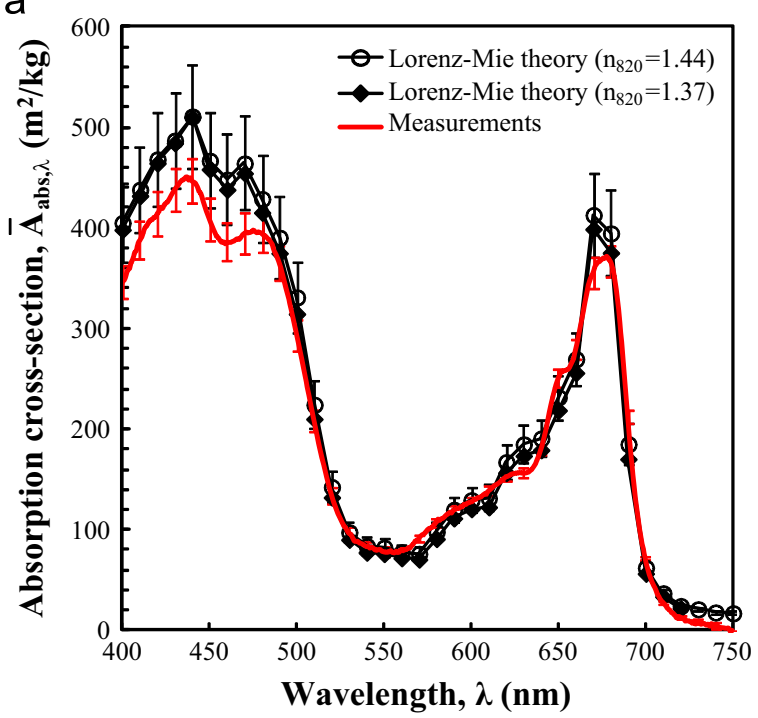

b

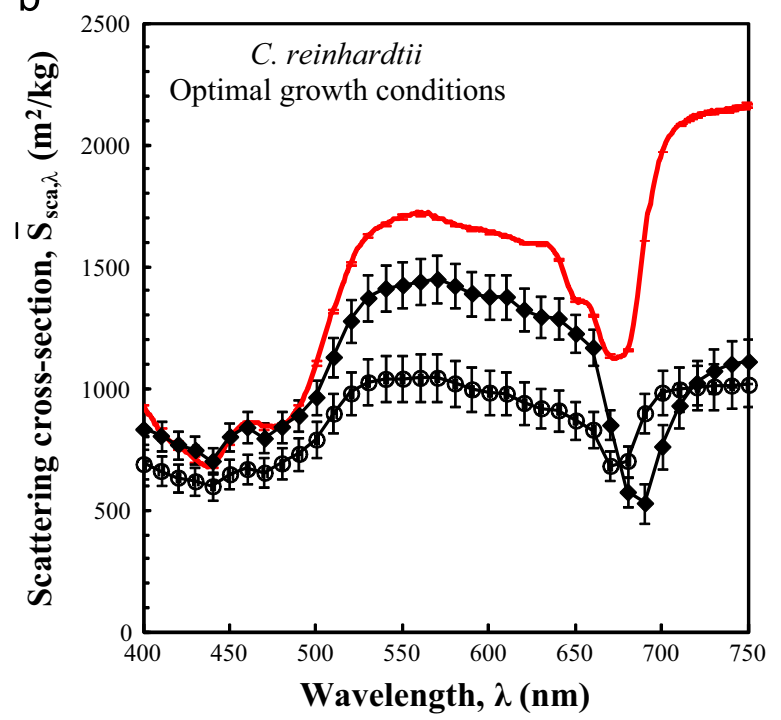

Fig. 4. Comparison between experimentally measured and theoretically predicted average spectral mass (a) absorption $\bar{A}_{a b s, \lambda}$ and (b) scattering $\bar{S}_{s c a, \lambda}$ cross-sections of $C$. reinhardtii grown in optimal conditions. Theoretical predictions treated cells as polydisperse spheres with $n_{820}$ equal to 1.44 or 1.37 .

pole expansion method [45], the multi-sphere T-matrix method [42,43], or the discrete-dipole-approximation (DDA) method [44] could be used to develop models that include various cell compartments in order to predict more accurately the microalgae scattering phase function $\Phi_{T, \lambda}$. However, for predicting the fluence rate and the rate of photon absorption in PBRs, with the objective of predicting and controlling the biochemical process, the integral properties such as the backward scattering ratio $b_{\lambda}$ are sufficient $[12,34]$. What's more, for such large scatterers, $b_{\lambda}$ is very small and has little effect on the fluence rate predicted by Eqs. (17) and (18).

Finally, the factor $\epsilon_{n}$ used for correcting the measured scattering cross-section of the microorganisms was determined according to Eq. (4) as 0.67 and 0.72 for $C$. reinhardtii grown in optimal conditions and in nitrogenlimited conditions, respectively, and 0.71 for C. vulgaris grown in optimal conditions.

\subsection{Absorption and scattering cross-sections}

\subsubsection{C. reinhardtii grown in optimal conditions}

Figs. 4(a) and (b) compare the experimentally measured and the theoretically predicted average spectral mass absorption $\bar{A}_{a b s, \lambda}$ and scattering $\bar{S}_{s c a, \lambda}$ cross-sections and their respective $95 \%$ confidence intervals for $C$. reinhardtii cultures grown in optimal conditions. Estimates of the absorption cross-section by either method were in relatively good agreement. In fact, the relative difference was well within the uncertainty of either method. The error bars in the theoretical predictions of the absorption cross-section were due to error propagations of the uncertainties in the measurements of the culture pigment and biomass concentrations and of the cell water mass fraction. The relative difference in $\bar{A}_{a b s, \lambda}$ between the two methods averaged over the PAR region was $8 \%$. In addition, the relative difference between the two methods for

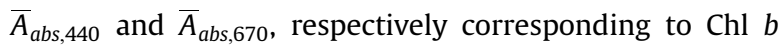
and $\mathrm{Chl} a$ absorption peaks, was smaller than $12 \%$ regardless of the anchor refractive index $n_{820}$. Note that the choice of anchor refractive index did not have a large influence on the theoretical predictions of $\bar{A}_{a b s, \lambda}$.

By contrast, the choice of anchor index of refraction $n_{820}$ had a large impact on the magnitude and shape of the spectral mass scattering cross-section $\bar{S}_{s c a, \lambda}$. Moreover, the PAR-averaged relative difference between the measured and predicted values of $\bar{S}_{s c a, \lambda}$ was large and around 33\% when the anchor refractive index $n_{820}$ was set to 1.44 . However, it diminished to $13 \%$ when the anchor refractive index $n_{820}$ was set to 1.37. Nevertheless, large discrepencies remained particularly for wavelengths larger than $650 \mathrm{~nm}$. This indicates the need for more advanced radiation models to accurately represent the non-spherical and heterogeneous nature of the microalgae cell. The multi-sphere T-matrix method $[42,43]$ or the DDA method [44] could be used to model the heterogeneity in the cells. Note, however, that several physical parameters must be determined for accurate representation of the cells including the spectral refractive index of the different cell compartments over the PAR region and their volume fraction and location within the cell as well as the cell water content [20]. Additionally, the anchor wavelength and refractive index must be chosen carefully in order to accurately predict the scattering cross-section of microalgae. However, the lack of information in the literature about the refractive index and the anchor wavelength of different microalgae species and strains may suggest that experimental measurements must always be performed before the theoretical method can be used to predict the radiation characteristics of each species or strain.

\subsubsection{C. reinhardtii grown in nitrogen-limited conditions}

Fig. 5(a) and (b) plot the experimentally measured and the theoretically predicted average spectral mass absorption $\bar{A}_{a b s, \lambda}$ and scattering $\bar{S}_{s c a, \lambda}$ cross-sections and their 

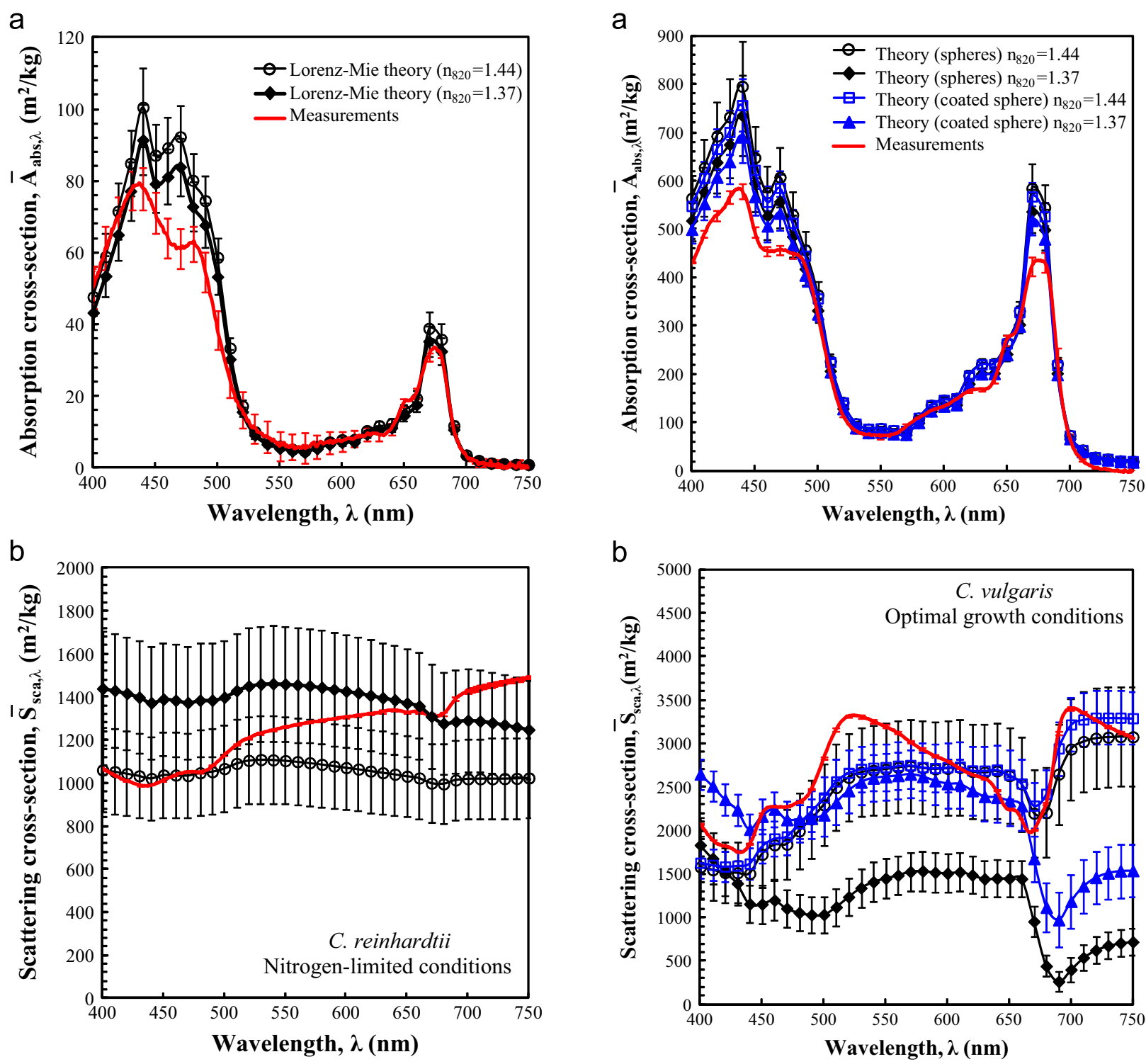

Fig. 5. Comparison between experimentally measured and theoretically predicted average spectral mass (a) absorption $\bar{A}_{a b s, \lambda}$ and (b) scattering $\bar{S}_{s c a, \lambda}$ cross-sections of $C$. reinhardtii grown in nitrogen-limited conditions. Theoretical predictions treated cells as polydisperse spheres with $n_{820}$ equal to 1.44 or 1.37 .

respective $95 \%$ confidence intervals for $C$. reinhardtii cultures grown in nitrogen-limited conditions. First, the average mass absorption cross-section $\bar{A}_{a b s, \lambda}$ of the nitrogenlimited $C$. reinhardtii cells were much smaller than that for cells grown under optimal conditions (Fig. 4(a)) due to their lower pigment concentrations (Table 1$)$. The predicted average spectral mass absorption cross-section $\bar{A}_{a b s, \lambda}$ agreed well with the measurements with a PAR-averaged relative difference of $16 \%$ and $14 \%$ when the anchor refractive index $n_{820}$ was 1.44 and 1.37 , respectively. However, the relative difference between the measured and predicted values of $\bar{A}_{a b s, \lambda}$ reached up to $53 \%$ between 450 and $550 \mathrm{~nm}$. Photosynthetic and photoprotective carotenoid pigments are responsible for absorption in this wavelength range. The

Fig. 6. Comparison between experimentally measured and theoretically predicted average spectral mass (a) absorption $\bar{A}_{a b s, \lambda}$ and (b) scattering $\bar{S}_{s c a, \lambda}$ cross-sections of $C$. vulgaris grown in optimal conditions. Theoretical predictions treated the cells as polydisperse spheres or coated spheres with $n_{820}$ equal to 1.44 or 1.37 .

differences in $\bar{A}_{a b s, \lambda}$ between the two methods may be due to inaccuracies in the measured carotenoid pigment concentration and/or to the inappropriate use of the spectral specific absorption cross-sections $E a_{P P C}$ and $E a_{P S C}$ of the carotenoid pigments used to predict the cell effective absorption index [Eq. (14)]. Indeed, the values of $E a_{P P C, \lambda}$ and $E a_{P S C, \lambda}$ used in the predictive method correspond to $\beta$-carotene and fucoxanthin, respectively. However, Krinsky and Levine [46] reported the major carotenoids in $C$. reinhardtii as $\beta$-carotene, violaxnthin, lutein, and neoxanthin. These carotenoid pigments may have different specific absorption cross-sections whose in vivo values have not been measured or reported in the literature [47]. Moreover, the types of carotenoids in the cells change in response to nutrient 
limitation [48]. Therefore, representing all of the photoprotective or photosynthetic carotenoids with a single absorption spectrum may be practical but may introduce inaccuracies in the predictions of cell absorption crosssections $\bar{A}_{a b s, \lambda}$. These discrepancies were more apparent in the nitrogen-limited $C$. reinhardtii culture as the cell carotenoid to chlorophyll ratio was nearly four times larger than in cells grown under optimal conditions.

Moreover, the PAR-averaged relative difference between the measured and the predicted scattering cross-section $\bar{S}_{s c a, \lambda}$ was $23 \%$ and $12 \%$ when the anchor refractive index $n_{820}$ was set to 1.37 and 1.44 , respectively. This indicates that for nitrogen-limited $C$. reinhardtii cells, the appropriate anchor refractive index $n_{820}$ was 1.44 compared with 1.37 for $C$. reinhardtii grown under optimal conditions. Comparison with previous results for optimal conditions suggests that the refractive index of the cells may vary with cultivation conditions as a result of changes in the biochemical composition of the cells. Therefore, the anchor refractive index must be known not only for each species but also for different operating conditions.

\subsubsection{C. vulgaris grown in optimal conditions}

Fig. 6(a) and (b) shows the experimentally measured and the theoretically predicted average spectral mass absorption $\bar{A}_{a b s, \lambda}$ and scattering $\bar{S}_{s c a, \lambda}$ cross-sections and their respective $95 \%$ confidence intervals for $C$. vulgaris cultures grown in optimal conditions. Unlike for $C$. reinhardtii, the theoretical predictions treating the cells as homogeneous spheres did not agree well with the measured average spectral mass absorption cross-section $\bar{A}_{a b s, \lambda}$ of the C. vulgaris cells, regardless of the anchor point selected. For example, the measured and predicted values of $\bar{A}_{a b s, \lambda}$ at $430 \mathrm{~nm}$, corresponding to one of Chl $a$ absorption peaks, were $580 \pm 15 \mathrm{~m}^{2} / \mathrm{kg}$ and $840 \pm 100 \mathrm{~m}^{2} / \mathrm{kg}$, respectively. This $43 \%$ difference corresponded to the largest relative difference in $\bar{A}_{a b s, \lambda}$ between the experimental and theoretical methods. In addition, the PAR-averaged relative difference in $\bar{A}_{a b s, \lambda}$ was equal to $22 \%$. Similarly, the PAR-averaged relative difference in the spectral mass scattering cross-section $\bar{S}_{s c a, \lambda}$ between the two methods was $13 \%$ and $49 \%$ when the anchor refractive index $n_{820}$ was 1.44 and 1.37 , respectively. The relatively large deviations in both $\bar{A}_{a b s, \lambda}$ and $\bar{S}_{s c a, \lambda}$ could not be attributed to uncertainties in the pigment and biomass concentrations or in the cell water content $x_{w}$. We speculate that these differences were due to the presence of a refracting $130 \mathrm{~nm}$ thick sporolinelin wall surrounding the cells [31] rendering the treatment of $C$. vulgaris as homogeneous spheres invalid.

Alternatively, C. vulgaris cells were modeled as coated spheres with an outer radius identical to that of the homogeneous sphere $r_{e q}$ (Fig. 2(d)). As previously stated, the wall was assumed to be non-absorbing but refracting with a refractive index $n_{w}$ equal to 1.48 [32]. Fig. 6 indicates that both the predicted spectral mass absorption $\bar{A}_{a b s, \lambda}$ and scattering $\bar{S}_{s c a, \lambda}$ cross-sections obtained by treating $C$. vulgaris as coated spheres agreed relatively well with experimental measurements. First, the PAR-averaged relative difference between the experimental and theoretical values of $\bar{A}_{a b s, \lambda}$ was $17 \%$ and $10 \%$ when the anchor refractive index was 1.44 and 1.37 , respectively. It is interesting to note that $\bar{A}_{a b s, \lambda}$ was smaller across the PAR region when treating the cells as coated spheres instead of homogeneous spheres despite having the same mass of pigments. This can be attributed to the so-called package effect [20]. In addition, the PAR-averaged relative difference in the average spectral mass scattering cross-section $\bar{S}_{s c a, \lambda}$ between experimental measurements and the coated sphere approximation was $11 \%$ and $19 \%$ when the anchor refractive index $n_{820}$ was 1.44 and 1.37 , respectively.

Finally, these results illustrate that some microorganisms cannot be modeled as homogeneous spheres when their cell wall is thick relative to their diameter and strongly refracting. Further studies should investigate the conditions under which spheroidal heterogeneous microalgae cells can be treated as homogeneous spheres. In this context, determining the cell wall thickness and the spectral optical properties of the various cell compartments or organelles is essential and currently lacking. Finally, it remains unclear if the chloroplast encasing the absorbing pigments needs to also be modeled as a separate entity inside the cell. For example, the chloroplast or the nucleus can be included in the cell model using the multi-pole expansion method [45] or the multi-sphere T-matrix method $[42,43]$. Note that these two methods are limited to modeling the cell and its constituents as spheres and require considerable computational resources.

\subsection{Normal-hemispherical transmittance}

Fig. 7(a) and (b) compare the measured and predicted normal-hemispherical transmittance $T_{n h, \lambda}$ spectra of $C$. reinhardtii grown in (a) optimal $\left(X=0.11 \mathrm{~kg} / \mathrm{m}^{3}\right)$ and (b) nitrogen-limited $\left(X=0.17 \mathrm{~kg} / \mathrm{m}^{3}\right)$ conditions. Similar comparison was performed by Dauchet et al. [13] to validate their theoretical predictions of the radiation characteristics of $C$. reinhardtii. Here, the cells were modeled as polydisperse homogeneous spheres with anchor refractive index $n_{820}=1.44$ or 1.37. Similarly, Fig. $7(\mathrm{c})$ and (d) compare the measured normal-hemispherical transmittance of $C$. vulgaris grown in optimal conditions $\left(X=0.56 \mathrm{~kg} / \mathrm{m}^{3}\right)$ and modeled as (c) homogeneous spheres and $(\mathrm{d})$ coated spheres representing the refracting cell wall with anchor refractive index $n_{820}=1.44$ or 1.37 . The normal-hemispherical transmittance $T_{n h, \lambda}$ was predicted using the modified two-flux approximation [Eqs. (21)(28)] employing either the experimentally measured or the theoretically predicted values of $\bar{A}_{a b s, \lambda}, \bar{S}_{s c a, \lambda}$, and $g_{\lambda}$. Note that the measurements of $T_{n h, \lambda}$ shown in Fig. 7 were not used for the determination of $\bar{A}_{a b s, \lambda}$ or $\bar{S}_{s c a, \lambda}$. Here, in fact, the biomass concentrations were relatively large and multiple scattering prevailed.

For all three cultures, excellent agreement was found between the experimentally measured normal-hemispherical transmittance $T_{n h, \lambda}$ and the predictions using the measured radiation characteristics. The relative error was smaller than $5 \%$ at any wavelength in the PAR region. This indicates that the modified two-flux approximation [Eq. (21)] is an adequate model to predict the normal-hemispherical transmittance $T_{n h, \lambda}$ when paired with the measured radiation characteristics of the microalgae. 
a

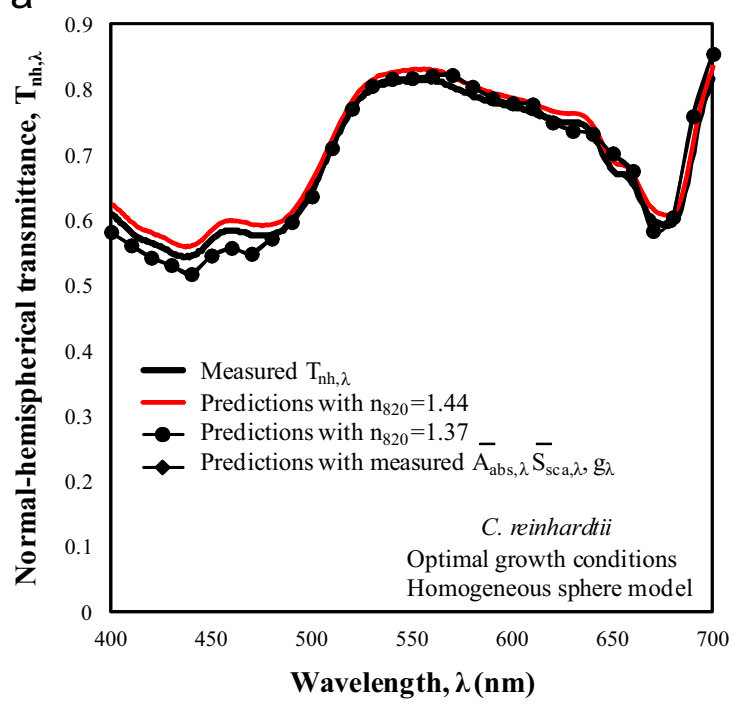

C

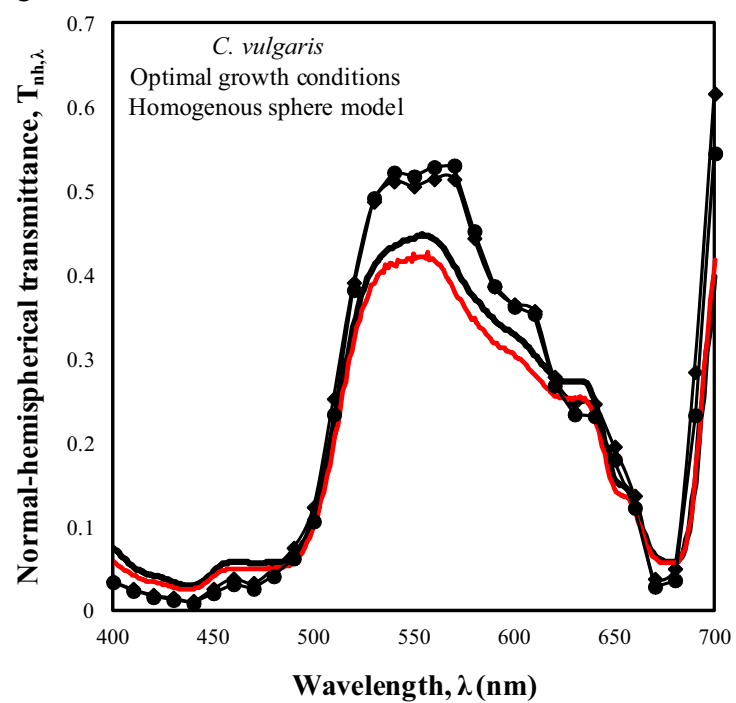

b

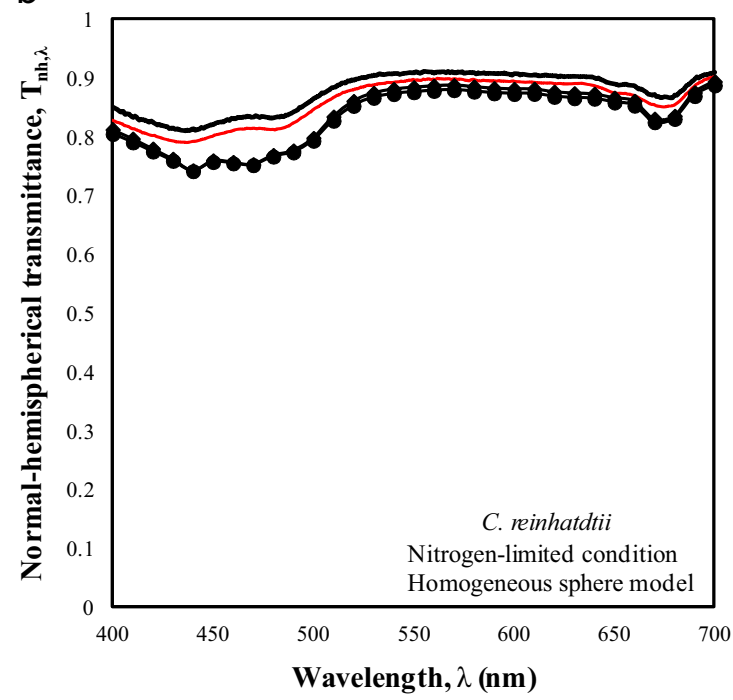

d

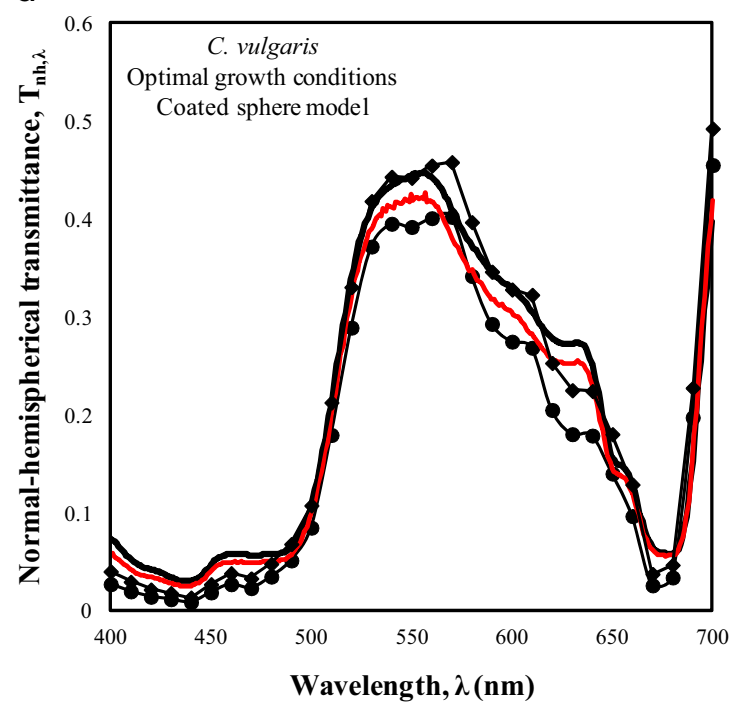

Fig. 7. Comparison of the measured and predicted normal-hemispherical transmittance $T_{n h, \lambda}$ of concentrated samples of (a) $C$. reinhardtii grown in optimal conditions $\left(X=0.11 \mathrm{~kg} / \mathrm{m}^{3}\right)$, (b) $C$. reinhardtii grown in nitrogen-limited conditions $\left(X=0.17 \mathrm{~kg} / \mathrm{m}^{3}\right)$, and $C$. vulgaris grown in optimal conditions $\left(X=0.56 \mathrm{~kg} / \mathrm{m}^{3}\right)$ using predicted radiation characteristics for (c) homogeneous sphere and (d) coated spheres. Predictions of transmittance $T_{n h, \lambda}$ was based on the modified two-flux approximation [Eq. (21)].

Moreover, the PAR-averaged relative error between the measured and the predicted values of $T_{n h, \lambda}$ using the theoretical radiation characteristics of $C$. reinhardtii grown in optimal conditions or in nitrogen-limited conditions was less than $7.5 \%$. The choice of anchor refractive index had no significant influence on the predictions of $T_{n h, \lambda}$. As previously discussed, the anchor refractive index had a significant effect on the prediction of the cells' scattering cross-section $\bar{S}_{s c a, \lambda}$. However, the latter did not have a measurable impact on the normal-hemispherical transmittance of the sample. This was due to the fact that (i) the microorganisms were strongly forward scattering with a large asymmetry factor such that $\bar{A}_{a b s, \lambda} \gg\left(1-g_{\lambda}\right) \bar{S}_{s c a, \lambda}$ and (ii) the light scattered by the suspension was collected by the integrating sphere when measuring $T_{n h, \lambda}$. In fact, such measurements are used to estimate the absorption coefficient of the microalgae suspension $\kappa_{\lambda}$ [Eq. (2)]. As a consequence, the validation performed by Dauchet et al. [13] and replicated here can only "validate" the prediction of cells' absorption cross-section and indirectly the model for the absorption index $k_{\lambda}$ [Eq. (14)]. However, it cannot precisely validate predictions of cells' scattering crosssection which depends strongly on the effective refractive index $n_{\lambda}$.

Lastly, predicting $T_{n h, \lambda}$ using Eq. (21) and the theoretical radiation characteristics predicted by the Lorenz-Mie theory for $C$. vulgaris treated as homogeneous spheres showed poor agreement with experimental data. However, treating $C$. vulgaris as coated spheres gave reasonably good agreement. This indicates that $C$. vulgaris cells are better 
C. reinhardtii optimal growth conditions
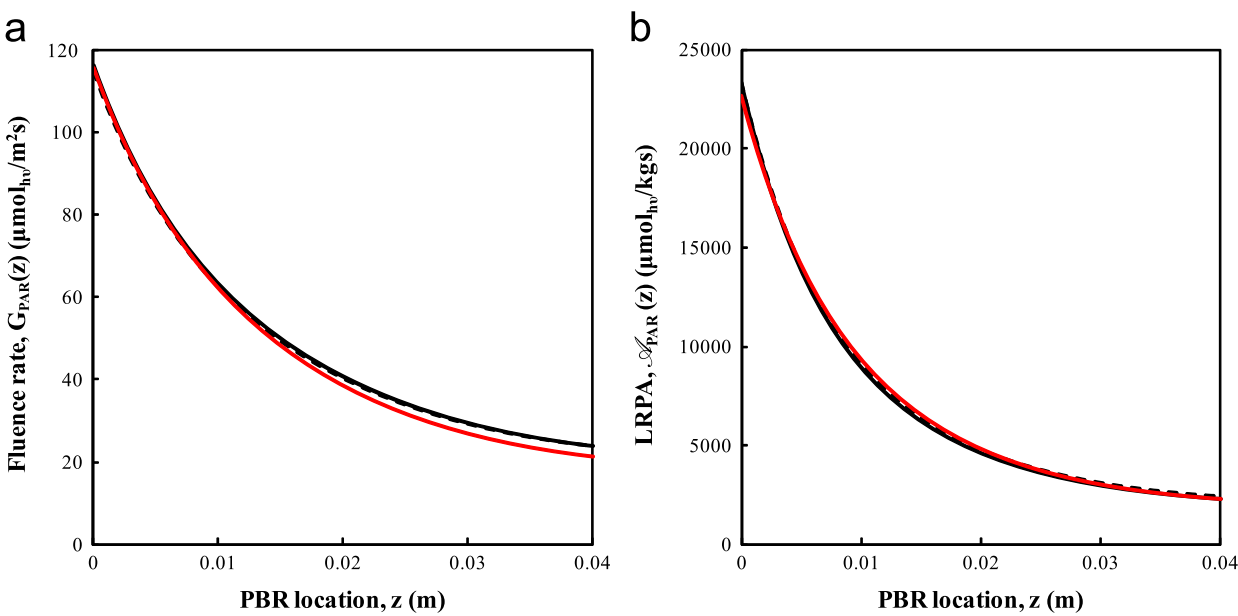

C. reinhardtii nitrogen-limited conditions

C

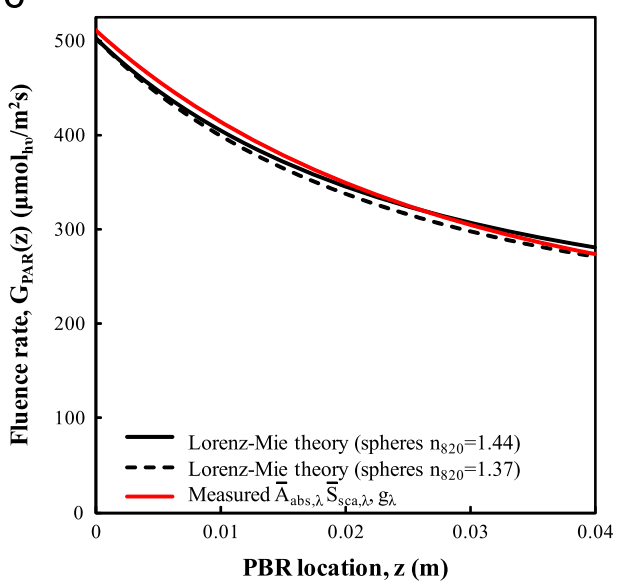

d

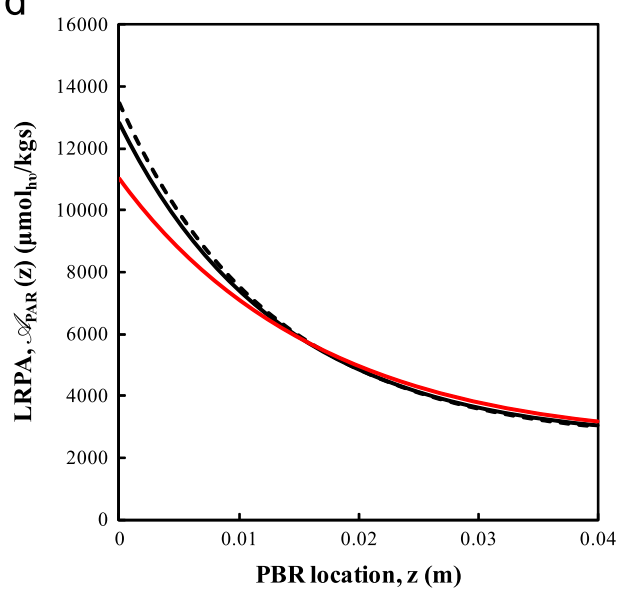

C. vulgaris optimal growth conditions

e

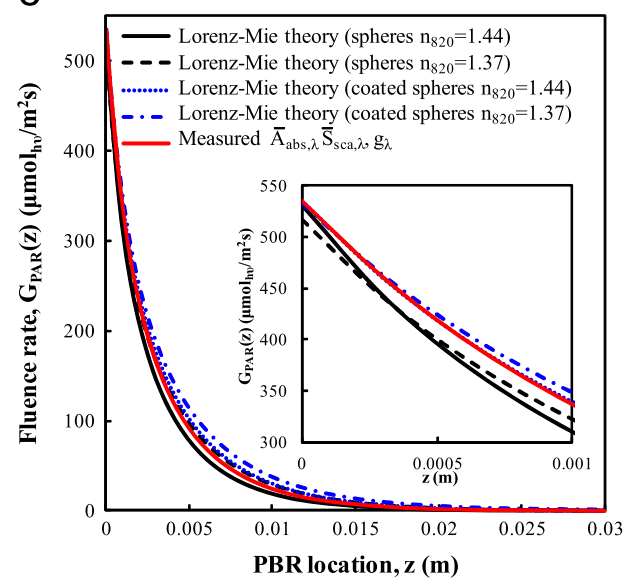

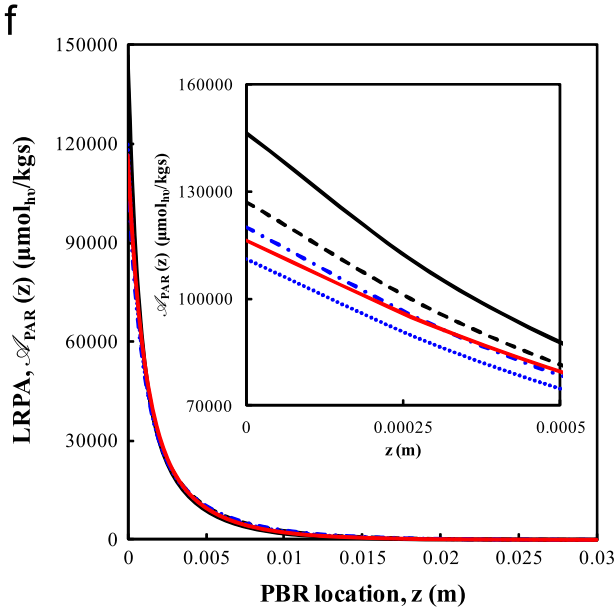

Fig. 8. PAR-averaged fluence rate $G_{P A R}(z)$ and local rate of photon absorption LRPA $\mathcal{A}(z)$ in the PBRs predicted by Eqs. (17) and (16) for (a-b) C. reinhardtii grown in optimal conditions $\left(X=0.35 \mathrm{~kg} / \mathrm{m}^{3}\right)$, (c-d) C. reinhardtii grown in nitrogen-limited conditions $\left(X=0.87 \mathrm{~kg} / \mathrm{m}^{3}\right)$, and (e-f) C. vulgaris grown in optimal conditions $\left(X=2.1 \mathrm{~kg} / \mathrm{m}^{3}\right)$ using measured and theoretically predicted radiation characteristics. 
represented as coated spheres than as homogeneous spheres. Here also, the anchor refractive index had a negligible effect on the PAR-averaged relative error in $T_{n h, \lambda}$.

\subsection{Fluence rate and the local rate of photon absorption}

Fig. 8(a)-(f) shows the PAR-averaged fluence rate $G_{P A R}(z)$ and local rate of photon absorption (LRPA) $\mathcal{A}_{P A R}(z)$ in the PBR, for all three cultures, estimated using Eqs. (19) and (20), respectively. Predictions were made using either the measured or theoretical radiation characteristics using anchor refractive index $n_{820}$ equal to 1.44 or 1.37 .

Experimentally, the $C$. reinhardtii culture grown under optimal conditions achieved a steady-state biomass concentration $X$ of $0.35 \mathrm{~kg} / \mathrm{m}^{3}$. At this concentration, the use of measured or theoretical radiation characteristics produced nearly identical predictions of the PAR-averaged fluence rate $G_{P A R}(z)$ (Fig. 8(a)) and LRPA $\mathcal{A}_{\text {PAR }}(z)$ (Fig. 8(b)) with a relative difference of less than $10 \%$ regardless of the anchor refractive index. For practical purposes, this difference is negligible and both experimental and theoretical predictions treating the cell as homogeneous sphere with effective optical properties given by Eqs. (14) and (15) are essentially equivalent. Indeed, we recently demonstrated that a depth-averaged relative error in fluence rate $G_{P A R}(z)$ of $15 \%$ resulted in relative error in biomass productivity predictions of less than $6 \%$ for C. vulgaris grown in a flat-plate torus PBR with pathlength $L=4 \mathrm{~cm}$ and biomass concentration $X=0.34 \mathrm{~kg} / \mathrm{m}^{3}$ exposed to $q_{\text {in }}^{\prime \prime}=200$ $\mu \mathrm{mol}_{h \nu} / \mathrm{m}^{2} \cdot \mathrm{s}$ of white LED lights [49].

Moreover, the $C$. reinhardtii culture grown in nitrogenlimited conditions had a steady-state biomass concentration $X$ equal to $0.87 \mathrm{~kg} / \mathrm{m}^{3}$. For this concentration, the fluence rate $G_{P A R}(z)$ estimated with the measured or the theoretical radiation characteristics had a relative difference of less than $4 \%$ regardless of the anchor refractive index $n_{820}$ used (Fig. 8(c)). On the other hand, the PARaveraged relative difference in LRPA estimated using the measured and theoretical radiation characteristics was $16 \%$ or $23 \%$ when anchor point refractive index was 1.44 or 1.37, respectively (Fig. $8(\mathrm{~d})$ ). These differences may be due to the overestimation in absorption cross-section between $450 \mathrm{~nm}$ and $500 \mathrm{~nm}$, as previously discussed. Nevertheless, the theoretical predictions can be used as a good first order approximation in order to obtain the LRPA in the PBR.

Finally, $C$. vulgaris cells grown in optimal conditions had a steady-state biomass concentration $X$ equal to $2.11 \mathrm{~kg} /$ $\mathrm{m}^{3}$. Here, $G_{P A R}(z)$ and $\mathcal{A}_{P A R}(z)$ estimated using the measured radiation characteristics matched closely with those estimated using the predicted radiation characteristics for the coated spheres with anchor point refractive index $n_{820}=1.44$.

\section{Conclusion}

This study directly compared state of the art experimental and theoretical methods for determining the radiation characteristics of microalgae. The theoretical method was based on the Lorenz-Mie theory treating microalgae as polydisperse volume-equivalent homogeneous spheres. It was combined with a model for the effective spectral complex index of refraction of the microalgae cells depending on the measured pigment concentrations and on the anchor refractive index. Relatively good agreement was found between the two methods for predicting the absorption and scattering cross-sections and the asymmetry factor and the backward scattering fraction of $C$. reinhardtii grown either in optimal or in nitrogen-limited conditions provided the anchor refractive index was properly chosen. However, the measured absorption cross-section of $C$. vulgaris grown in optimal conditions did not agree with theoretical predictions treating the cells as volume-equivalent homogeneous spheres. Instead, modeling $C$. vulgaris cells as coated spheres with a non-absorbing outer shell, representing their thick refracting cell wall made of sporolinelin [31], gave good predictions of the integral radiation characteristics. The results also indicate the need to develop more sophisticated theoretical models in order to more accurately predict the radiation characteristics of microalgae, in particular the scattering phase function. In particular, the models should account for cell heterogeneity and non-sphericity. However, models based on the Lorenz-Mie theory, explored in the study, approximating the microalgae as homogeneous or coated spheres present the advantage of being simple and fast while predicting radiation characteristics that are sufficiently accurate to estimate the relevant parameters for photobiological processes, namely the fluence rate and the local rate of photon absorption.

\section{Acknowledgements}

The project was supported by French National Research Agency project DIESALG (ANR-12-BIME-0001-02). L.P. is grateful to the Région Pays de la Loire for the Research Chair for International Junior Scientists. We thank Dr. Jérémy Dauchet for useful discussion and exchange of information.

\section{References}

[1] Borowitzka MA. High-value products from microalgae-their development and commercialisation. J Appl Phycol 2013;25(3):743-56.

[2] Moody JW, McGinty CM, Quinn JC. Global evaluation of biofuel potential from microalgae. Proc Natl Acad Sci 2014;111(23):8691-6.

[3] Cornet J-F, Dussap CG, Cluzel P, Dubertret G. A structured model for simulation of cultures of the cyanobacterium Spirulina platensis in photobioreactors: II. Identification of kinetic parameters under light and mineral limitations. Biotechnol Bioeng 1992;40:826-34.

[4] Molina-Grima E, Camacho FG, Sánchez-Pérez JA, Fernández-Sevilla JM, Acién-Fernández FG, Contreras-Gómez A. A mathematical model of microalgal growth in light-limited chemostat culture. J Chem Technol Biotechnol 1994;61(2):167-73.

[5] Cornet JF, Dussap CG. A simple and reliable formula for assessment of maximum volumetric productivities in photobioreactors. Biotechnol Progress 2009;25:424-35.

[6] Takache H, Christophe G, Cornet J-F, Pruvost J. Experimental and theoretical assessment of maximum productivities for the microalgae Chlamydomonas reinhardtii in two different geometries of photobioreactors. Biotechnol Progress 2010;26(2):431-40. 
[7] Takache H, Pruvost J, Cornet J-F. Kinetic modeling of the photosynthetic growth of Chlamydomonas reinhardtii in a photobioreactor. Biotechnol Progress 2012;28(3):681-92.

[8] Béchet Q, Shilton A, Guieysse B. Modeling the effects of light and temperature on algae growth: state of the art and critical assessment for productivity prediction during outdoor cultivation. Biotechnol Adv 2013;31(8):1648-63.

[9] Cornet J-F, Dussap CG, Dubertret G. A structured model for simulation of cultures of the cyanobacterium Spirulina platensis in photobioreactor: I. Coupling between light transfer and growth kinetics. Biotechnol Bioeng 1992;40:817-25.

[10] Pilon L, Berberoğlu H, Kandilian R. Radiation transfer in photobiological carbon dioxide fixation and fuel production by microalgae. J Quant Spectrosc Radiat Transf 2011;112(17):2639-60.

[11] Pilon L, Kandilian R. Interaction between light and photosynthetic microorganisms. In: Legrand J, editor. Thematic issue on photobioreaction engineering. Advances in chemical engineering, vol. 4. The Netherlands: Elsevier; 2015.

[12] Pottier L, Pruvost J, Deremetz J, Cornet J-F, Legrand J, Dussap CG. A fully predictive model for one-dimensional light attenuation by Chlamydomonas reinhardtii in a torous photobioreactor. Biotechnol Bioeng 2005;91:569-82.

[13] Dauchet J, Blanco S, Cornet J-F, Fournier R. Calculation of the radiative properties of photosynthetic microorganisms. J Quant Spectrosc Radiat Transf 2015;161:60-84.

[14] Pruvost J, Van Vooren G, Cogne G, Legrand J. Investigation of biomass and lipids production with Neochloris oleoabundans in photobioreactor. Bioresour Technol 2009;100(23):5988-95.

[15] Van Vooren G, Le Grand F, Legrand J, Cuine S, Peltier G, Pruvost J. Investigation of fatty acids accumulation in Nannochloropsis oculata for biodiesel application. Bioresour. Technol. 2012;124:421-32.

[16] Kandilian R, Lee E, Pilon L. Radiation and optical properties of Nannochloropsis oculata grown under different irradiances and spectra. Bioresour Technol 2013;137:63-73.

[17] Kandilian R, Pruvost J, Legrand J, Pilon L. Influence of light absorption rate by Nannochloropsis oculata on triglyceride production during nitrogen starvation. Bioresour Technol 2014;163:308-19.

[18] Heng R-L, Pilon L. Time-dependent radiation characteristics of Nannochloropsis oculata during batch culture. J Quant Spectrosc Radiat Transf 2014;144:154-63.

[19] Berberoğlu H, Yin J, Pilon L. Simulating light transfer in a bubble sparged photobioreactor for simultaneous hydrogen fuel production and $\mathrm{CO}_{2}$ mitigation. Int J Hydrog Energy 2007;32:2273-85.

[20] Jonasz M, Fournier G. Light scattering by particles in water: theoretical and experimental foundations. San Diego, CA: Academic Press; 2007.

[21] Kandilian R, Heng R-L, Pilon L. Absorption and scattering by fractal aggregates and by their equivalent coated spheres. J Quant Spectrosc Radiat Transf 2015;151:310-26.

[22] Heng R-L, Sy KC, Pilon L. Absorption and scattering by bispheres, quadspheres, and circular rings of spheres and their equivalent coated spheres. J Opt Soc Am A 2014;32(1):46-60.

[23] Lee E, Pilon L. Absorption and scattering by long and randomly oriented linear chains of spheres. J Opt Soc Am A 2013;30(9): 1892-900.

[24] Berberoğlu H, Pilon L, Melis A. Radiation characteristics of Chlamydomonas reinhardtii CC125 and its truncated chlorophyll antenna transformants tla1, tlaX, and tla1-CW. Int J Hydrogen Energy 2008;33(22):6467-83.

[25] Berberoğlu H, Yin J, Pilon L. Light transfer in bubble sparged photobioreactors for $\mathrm{H}_{2}$ production and $\mathrm{CO}_{2}$ mitigation. Int J Hydrog Energy 2007;32(13):2273-85.

[26] Kandilian R, Tsao T-C, Pilon L. Control of incident irradiance on a batch operated flat-plate photobioreactor. Chem Eng Sci 2014;119: 99-108.

[27] Modest MF. Radiative heat transfer, 3rd ed. Oxford, UK: Elsevier; 2013.
[28] Bidigare RR, Ondrusek MEE, Morrow JHH, Kiefer DAA. In-vivo absorption properties of algal pigments. Proc SPIE 1990;1302: 290-302.

[29] Lee E, Heng R-L, Pilon L. Spectral optical properties of selected photosynthetic microalgae producing biofuels. J Quant Spectrosc Radiat Transf 2013;114:122-35.

[30] Quirantes A, Bernard S. Light-scattering methods for modeling algal particles as a collection of coated and/or nonspherical scatterers. J Quant Spectrosc Radiat Transf 2006;100(1-3):315-24.

[31] Geken HG, Donohoe B, Knoshaug EP. Enzymatic cell wall degradation of Chlorella vulgaris and other microalgae for biofuels production. Planta 2013;237(1):239-53.

[32] Atkinson AW, Gunning BES, John PCL. Sporopollenin in the cell wall of Chlorella and other alga: ultrastructure, chemistry, and incorporation of 14C-acetate, studied in synchronous cultures. Planta 1972;107(1):1-32.

[33] Traverse A. Paleopalynology. Dordrecht, The Netherlands: Springer; 2007.

[34] Lee E, Pruvost J, He X, Ramakanth R, Pilon L. Design tool and guidelines for outdoor photobioreactors. Chem Eng Sci 2014;106: $18-29$.

[35] Dombrovsky LA, Randrianalisoa J, Baillis D, Pilon L. Use of Mie theory to analyze experimental data to identify infrared properties of fused quartz containing bubbles. Appl Opt 2005;44(33):7021-31.

[36] Ritchie RJ. Consistent sets of spectrophotometric Chlorophyll equations for acetone, methanol and ethanol solvents. Photosynth Res 2006;89(1):27-41.

[37] Strickland JDH, Parsons TR. A practical handbook of seawater analysis: pigment analysis, bulletin of fisheries research board of Canada. Fisheries Research Board of Canada; 1968.

[38] Dubinsky Z, Stambler N. Photoacclimation processes in phytoplankto: mechanisms, consequences, and applications. Aquat Microb Ecol 2000;56:163-76.

[39] Van De Hulst HC. Light scattering by small particles. New York, NY: Wiley; 1957.

[40] Souliés A, Pruvost J, Legrand J, Castelain C, Burghelea TI. Rheological properties of suspensions of the green microalga Chlorella vulgaris at various volume fractions. Rheol Acta 2013;52(6):589-605.

[41] Heraeus, 2015. Quartz glass for optics: Data and properties, http:// heraeus-quarzglas.com/media/webmedia_local/downloads/brosch ren_mo/DataandProperties_Optics_fusedsilica.pdf.

[42] Mishchenko MI, Liu L, Mackowski DW, Cairns B, Videen G. Multiple scattering by random particulate medi: exact 3d results. Opt Express 2007;15(6):2822-36

[43] Mishchenko MI, Liu L, Mackowski DW. Morphology-dependent resonances of spherical droplets with numerous microscopic inclusions. Opt Lett 2014;39(6):1701-4.

[44] Yurkin MA, Hoekstra AG. The discrete-dipole-approximation code add: capabilities and known limitations. J Quant Spectrosc Radiat Transf 2011;112(13):2234-47.

[45] Videen G, Ngo D. Light scattering multipole solution for a cell. J Biomed Opt 1998;3(2):212-20.

[46] Krinsky NI, Levine RP. Carotenoids of wild type and mutant strains of the green alga, Chlamydomonas reinhardtii. Plant Physiol 1964;39 (4):680-7.

[47] Rodriguez-Amaya DB. A guide to carotenoid analysis in foods. Washington D.C.: ILSI Press; 2001.

[48] Francis GW, Strand LP, Lien T, Knutsen G. Variations in the carotenoid content of Chlamydomonas reinhardtii throughout the cell cycle. Arch Microbiol 1975;104(1):249-54.

[49] Kandilian R, Soulies A, Rousseau B, Pruvost J, Legrand J, Pilon L. Simple method to measure spectral absorption cross-section of microalgae. Chem Eng Sci 2016, accepted. 